
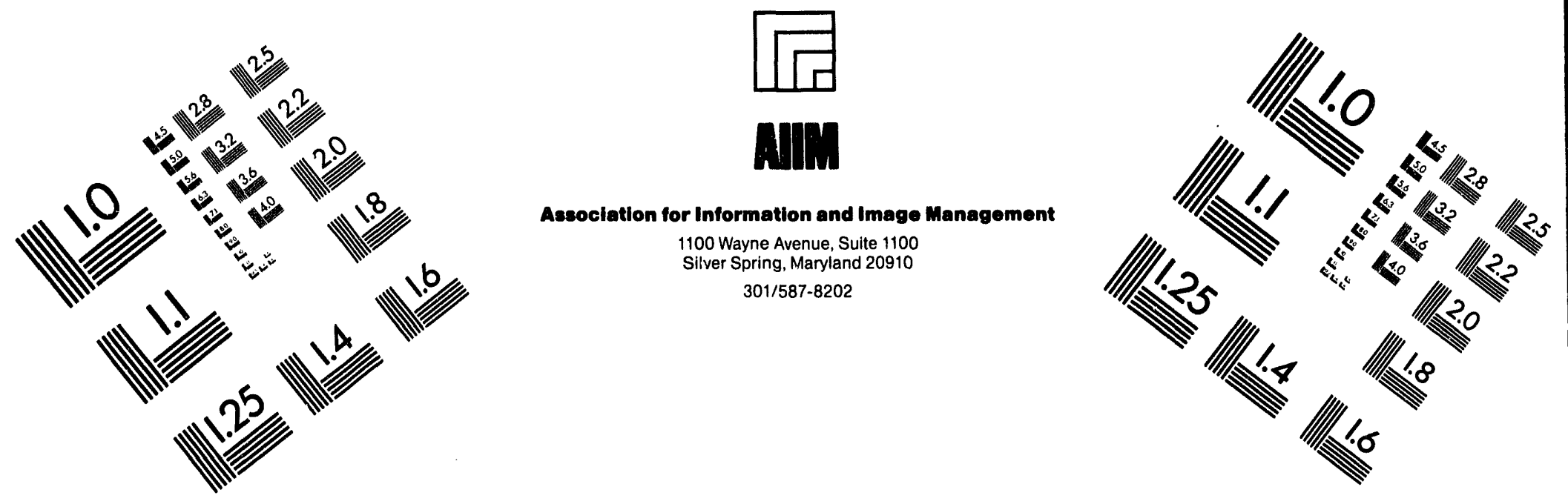

\title{
Centimeter
}

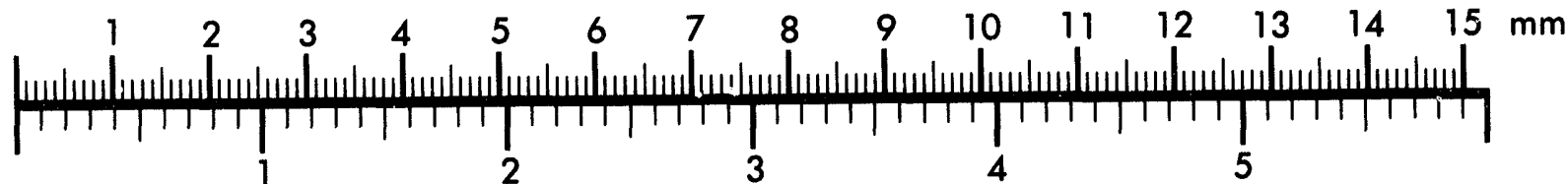

Inches
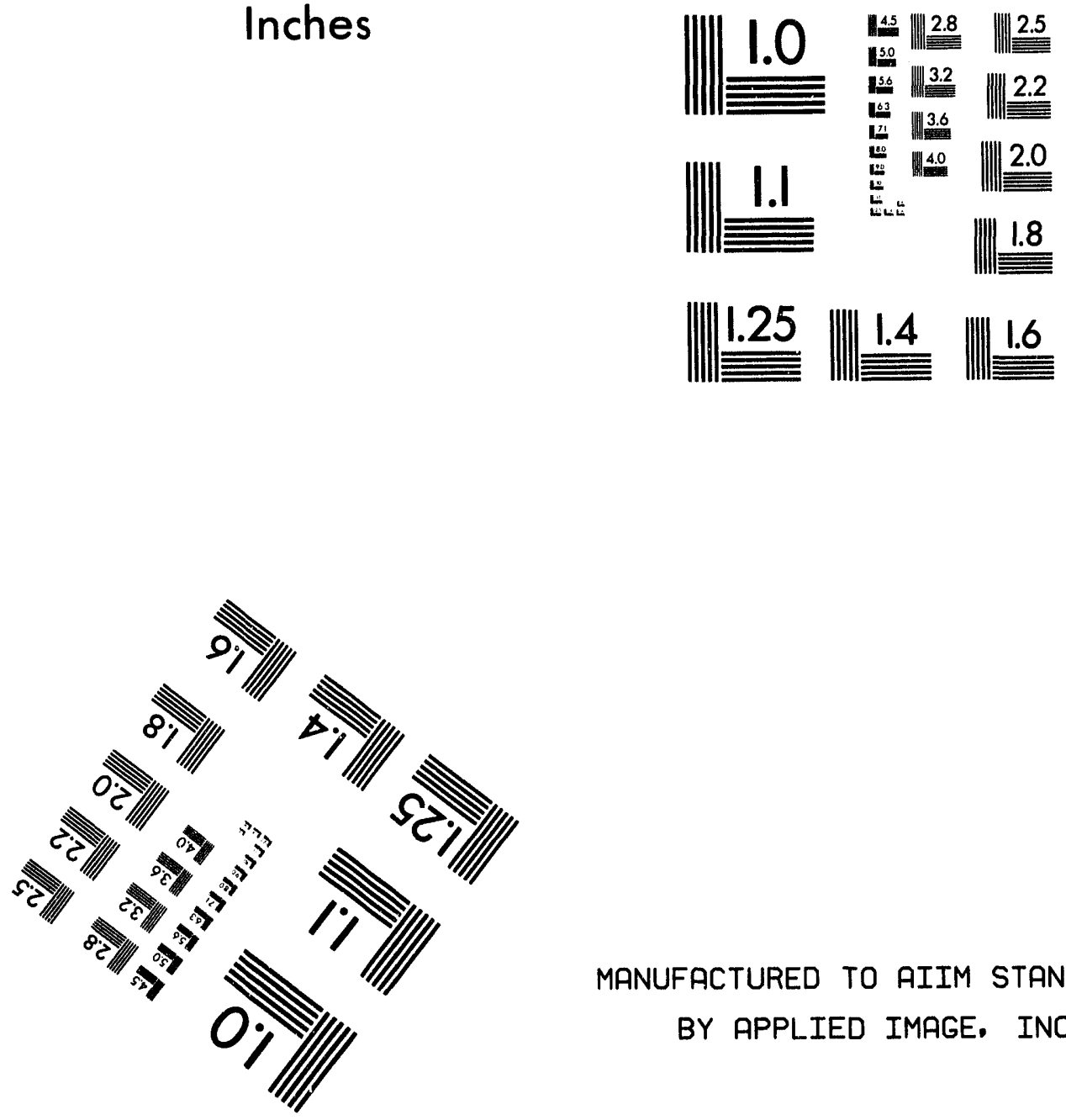

MANUFACTURED TO AIIM STANDARDS

BY APPLIED IMAGE, INC.

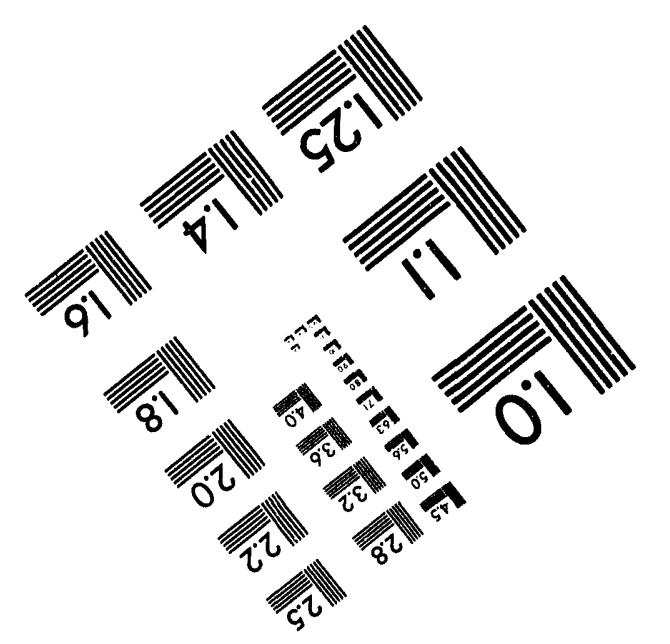



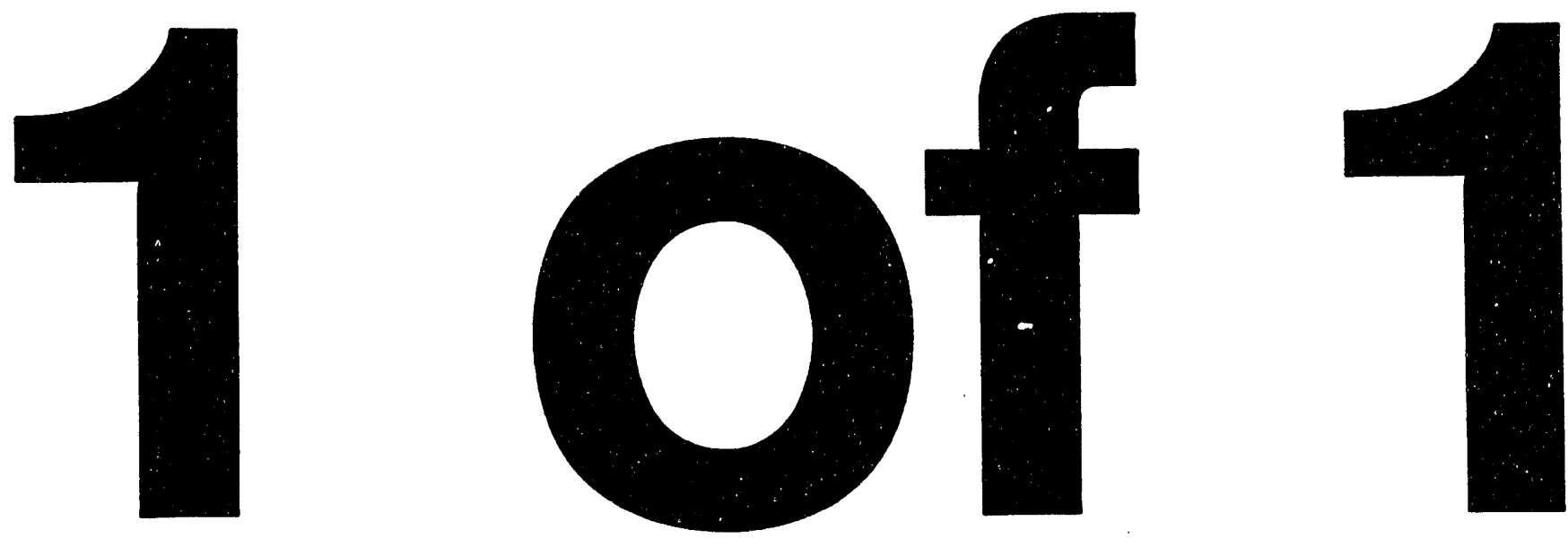
A New MCNPTM Test Set

Ronald C. Brockhoff

John S. Hendricks 


\title{
A NEW MCNP ${ }^{\mathrm{TM}}$ TEST SET
}

\author{
by \\ Ronald C. Brockhoff and John S. Hendricks
}

\begin{abstract}
The MCNP ${ }^{1}$ test set is used to test the MCNP code after installation on various computer plat forms. For MCNP4 and MCNP4A this test set included 25 test problems designed to test as many features of the MCNP code as possible. A new and better test set has been devised to increase coverage of the code from $85 \%$ to $97 \%$ with 28 problems. The new test set is as fast as and shorter than the MCNP4A test set. We describe the methodology for devising the new test set, the features that were not covered in the MCNP4A test set, and the changes in the MCNP4A test set that have been made for MCNP4B and its developmental versions. Finally, new bugs uncovered by the new test set and a compilation of all known MCNP4A bugs are presented.
\end{abstract}

MCNP is a trademark of the Regents of the University of California, Los Alamos National Laboratory. 


\section{MCNP4A Test Set Analysis}

\section{A. Background}

MCNP4A was released internationally to the Radiation Shielding and Information Center on October 1, 1993, along with a 25-problem test set. The test set consists of 25 INP input files, 25 MCTAL files which contain tally and tracking information in an ascii format that does not change from one MCNP version to another, and 25 output files which contain the complete MCNP output which usually changes from one MCNP version to another. The test set also contains a RUNPROB script to run the test problems, an XSDIR cross-section directory and TESTLIB1, the neutron, photon, and electron data libraries necessary for the test problems.

The MCNP4A test set is fast and portable. However, the MCNP4A test set could not use ENDF/B- $V^{2}$ neutron cross-section data because of export restrictions which were not lifted until April, 1994. The MCNP4A test set also could not use the final ENDF/B-VI ${ }^{3}$ data which were not available until October 1994. Therefore, the MCNP4A test set used mostly undistributed ENDL90 neutron cross-section data and some preliminary ENDF/B-VI neutron cross-section data.

Furthermore, we had no idea of what and how much of MCNP was tested by the test set. The MCNP4A test set was built upon the MCNP4.2 test set which was an unscientifically collected set of difficult problems. These problems were modified throughout the development of MCNP4A by adding new options as new features or bug corrections were added to MCNP4A. Also, an attempt was made to use all the MCNP input cards. But there was no way to test all combinations of all features of MCNP, and we had no idea how much of the code was not utilized by the test set.

\section{B. Preliminary Analysis}

Attempts to improve the test set by using available code performance software were disappointing. As a first cut the PROF and PROFVIEW ${ }^{4}$ software analysis packages were used to determine which MCNP subroutines were unused. A number of major MCNP features not tested by the test set were identified. However, this number was insufficient to determine which combinations of features were untested and we clearly needed to know which lines of MCNP were untested, not just which subroutines. Existing software was inadequate for the job. Therefore, a new approach was required. Analysis of MCNP was achieved by modifying every line of the code. These approaches and their results are described in the following sections.

A complete listing of all untested parts of MCNP4A and the remaining untested parts of MCNP with the new test set has been compiled and is included. Note that the MCNP geometry and tally plotting routines are specifically excluded from this study.

\section{Review of Subroutine Test Coverage}

As a first cut the PROF and PROFVIEW 4 software analysis packages were used to determine which MCNP subroutines were unused. The major untested features identified were: 
- type 19 fission;

- lost particles;

- adjoint calculations;

- generation of adjoint importances;

- KCODE problems with more than 10 active cycles;

- multigroup photons;

- some PRDMP options;

- spherically symmetric surface sources.

Because PROF and PROFVIEW only provided information on which routines of the MCNP code were entered and not detailed information on the lines of each routine that were executed, a more detailed look at essential features and combinations of features was needed.

\section{Inadequacies of Other Test Coverage Software}

After checking with the LANL CIC-Division consulting office and several hardware vendors, it appeared that DBX on the IBM cluster, with the trace option enabled, was the most promising means of identifying which lines of MCNP are currently tested by the MCNP4A test set. In theory, this program should produce a listing of the lines in the MCNP code that were executed using the test problems. However, a DBX trace of the MCNP code could not be completed successfully due to the complexity and length of MCNP. The DBX trace would discontinue without warning if complex lines were entered, and traces of greater than 300 Megs could not be made.

\section{E. A Successful "Brute Force" Approach}

To overcome the problems associated with the DBX trace, a program was written that would place a write statement after every line of the MCNP source code. This method allowed a trace to be performed that would not get lost in complex sections of the code. Unfortunately, the trace for a full MCNP run takes a long time using this method, and the resulting traces are several Gigabytes in size. Therefore, the program was modified to write to an array after each line of the MCNP source code to keep track of which lines were entered. Then at the end of the code, the resulting array are printed to a file. This technique produces a compact analysis of the lines of the MCNP code that are entered, and only requires a minimal increase in the computational time required to run the entire test set.

Using this "brute force" array analysis approach for analyzing the MCNP code, test set, and the associated libraries, several features and numerous combinations of features of the code were found to be untested. These features are categorized below: 


\section{F. Untested MCNP Features and Combinations}

In the following description of the untested features of MCNP4A, all of the labels refer to Fortran statement labels in the source code for MCNP4A.

\section{Geometry}

- The test problems do not test $\mathrm{K} / \mathrm{X}$ and $\mathrm{K} / \mathrm{Z}$ cones as indicated by the inactivity in label 180 of CHKCEL, labels 120-150 of ANGLE, and labels 160-190 of TRACK.

- The test problems do not test $\mathrm{KX}, \mathrm{KY}$, and $\mathrm{KZ}$ two-legged cones as indicated by the inactivity of label 230 in CHKCEL.

- Repeated structure tallies in lattices with single entry or triple entry constructs are untested as indicated by the inactivity of labels 72-79 and 91-94 in ITALLY.

- The plane, sphere, SQ surface, parabola, and two sheet cone specified by points are not tested as indicated by the inactivity of PSURF.

- The TR surface transformation card, where the rotation matrix is defined by a single vector, is not tested as indicated by inactivity in labels 30-80 in TRFMAT. In addition, the option of specifying one vector in each system is not tested as indicated by inactivity in labels $160-220$ in TRFMAT.

- A skew transformation of a surface resulting in a GQ general quadratic was not generated in any of the problems as indicated by inactivity in labels 50-60, and 100-120 of TRFSRF. In the test set there is only one skew transformation (TR5 in test problem 14) and that is applied to planes only. In addition, an orthogonal transformation of a torus is not tested as indicated by inactivity in labels 180-200 of TRFSRF.

- None of the problems require column input cell or surface area cards to be reordered as indicated by inactivity in labels $190-240$ in COLINP.

- Overflow of the LAJ array (adding a list of other-side cells) was not tested as indicated by inactivity in labels $70-90,180$, and 350 in NEWCEL.

- Electron problems do not contain spheres, cylinders except $\mathrm{CY}$, or cones as indicated by inactivity in label 30-210 in DBMIN, except for label 110 .

- None of the test problems test a general quadratic that degenerates to a sphere as indicated by inactivity in labels $120-140$ in AXIS. In addition, none of the test problems contain a torus that completely degenerates to a ellipsoid as indicated in labels 150-160 of OLDCRD.

- The test problems do not test continuations of the LIKE BUT cell card as indicated by inactivity in label 60 of LIKEBT. In addition, long rewrites of the LIKE BUT cell card are not tested as indicated by inactivity in label 90 and 180 of LIKEBT. Long rewrites can be tested by having a long continued LIKE BUT cell card in one of the test problems. 
- The LIKE BUT card is not used in unison with a ELPT:n card as indicated by inactivity in label 130 of LIKEBT. In addition, the LIKE BUT card is not used with MAT or RHO in any of the test problems as indicated by inactivity in label 140 of LIKEBT.

- A periodic boundary is not tested with a $\mathrm{P}$ plane as indicated by inactivity in label 80 of IPBC.

\section{Sources}

- Surface source continues are not tested as indicated by the inactivity of label 20 in SFILES.

- Options 4 (Gaussian), 5 (Evaporation), and 6 (Modified Gaussian) on the SP card are not tested as indicated by inactivity in labels $120-150$ in SMPSRC, and labels 100-180 in SPROB. In addition, some features of the option -21 (the power law) are not tested as indicated by inactivity in label 190 .

- User supplied sources are not tested as indicated by the inactivity of the subroutine CHKSRC, SOURCE, and SRCDX.

- No criticality source points in the lower level of a lattice are tested as indicated by the inactivity of label 80 in COLIDK.

- An initial criticality source overrunning itself is not tested as indicated by inactivity in label 200 in KCALC.

- The equiprobable bins (i.e., the SI card used in conjunction with the SP card with the default distribution) is not tested as indicated by inactivity in SPEC.

- Tabular energy distributions are not trimmed as indicated by inactivity in labels 10-80 in NORMA, and NORMH. To test this feature, we need to have an SI card with an energy range exceeding the energy range of the problem as indicated on the CUT and PHYS cards.

- Less than 30 active KCODE cycles were run in the test problems as indicated by inactivity in labels 200-240 of KNORM, labels 180-190, 230-260, and 320-330 of KPRINT, labels 20-30, and 140-150 in PRPLOT, inactivity in $\mathrm{KBATCH}$, and inactivity in KSKCYC.

- Spherically symmetric spherical surface sources are not tested as indicated by inactivity in labels 580-600 of OLDCRD, labels 170-200 in SURSRC, and inactivity in SSMSRC. To test this option we need a SYM=1 option on the SSW card.

- the SP card with the V option is not used as indicated in the "DO 300 LOOP" of VOLUME.

- Splitting and roulette on different numbers of particles in an RSSA run (i.e., the case of SSW and SSR with different NPS values) are not tested as indicated by inactivity in labels $130-160$ in SURSRC. 
- A standard source distribution such as $\mathrm{SPn}-\mathrm{m}$ is not tested with a limited energy range on the SI card as indicated by inactivity in labels $160-180$ in EQPBBN.

- Cell and surface flagging (CF, SF) is not used in a surface source (SSR) problem as indicated by inactivity in labels 10-30 in YPBSSP.

- Weight windows (WDWN, WWE, and WWN) are not used in a surface source (SSR) problem as indicated by inactivity in labels $60-120$ in YPBSSP.

- A unskewed spheroid source is not tested in any of the problems as indicated by inactivity in labels $330-340$ in SOURCB.

\section{Tallies}

- The FM card with parentheses is not tested as indicated by inactivity in ITALLY and the "DO 120 LOOP" in WTMULT.

- FM cards with line-of-sight attenuators are not tested as indicated by the inactivity of labels 280-410 in ITALPR, the "DO 140 LOOP" in STUFF, labels 230-270 in BINLIN, labels 220-260 in WTMULT, and label 150 of NXTIT1.

- Detailed photon production by reaction is not tested as indicated by the inactivity of labels $40-130,250,300-320$, and 380-410 in TPEFIL. This problem can be corrected by using ENDF/B-V or ENDF/B-VI cross sections in a MODE $\mathrm{N} P$ problem.

- Gaussian energy broadening used in conjunction with the pulse height tally F8 (i.e., FT card GEB option) is not tested as indicated by the inactivity of label 10 in FINPHT and label 280 in TALLYD.

- Point detectors or DXTRAN are not used with repeated structures or lattice geometries as indicated by inactivity in labels 10-20 in DDDET and inactivity in DDDLEV.

- Log combinations of the DE and DF cards are not tested, and the problems never test for particle energies below the lowest value on the $\mathrm{DE}$ card as indicated by inactivity in labels $30-60$ and 90 of DOSEF.

- Dosimetry libraries like 92235.59Y are not used for materials listed on the FM card as indicated by inactivity in labels 10-20 of GETXS.

- DXTRAN contributions are not made from free-gas thermal collisions as indicated by inactivity in labels 50-70 in DXTRAN. To test this feature, the energy cutoff would need to be lowered.

- Coincident detectors are not tested as indicated by inactivity in labels 80 and 100-120 of TALLOC and labels 160 and 200 of TALLYD.

- Time convolution is not done with cell tallies as indicated by inactivity in label 510 of TALLY. This feature should be tested with the F4 tally in a test problem. 
- Time convolution (i.e., FT TMC) is not tested with a detector tally as indicated by inactivity in labels $310-330$ and 470 of TALLYD.

- DXTRAN is not tested with the S or Q option on the SI or SP card as indicated by inactivity in label 460 of CALCPS.

- Pulse height tallies are not tested with repeated structures/lattices tallying on a single lattice element or structure as indicated by inactivity in labels 10-25 of TALPH.

\section{Physics}

- Neutron analog capture is not used in a criticality problem as indicated by the inactivity of label 115 in COLIDN.

- The free gas thermal treatment does not adjust the cross sections on the fly, probably because the temperature is constant in most problems as indicated by the inactivity of labels 140-150 in ACETOT. A time-dependent temperature needs to be tested. Test problem 2 has time-dependent temperatures, but they are never tested because of a $1 \mathrm{KeV}$ energy cutoff. I recommend lowering the energy cutoff in this problem to zero and testing the higher energy cutoff in some other problem.

- The $S(\alpha, \beta)$ thermal treatment did not adjust the elastic cross sections because JXS $(4)$ from the $S(\alpha, \beta)$ thermal libraries was always zero as indicated by the inactivity of labels 200-250 in ACETOT : nd labels 10,60-70, and 90-120 of SABCOL. This feature can only be tested if we use an $S(\alpha, \beta)$ with JXS(4) not equal to zero in some problem.

- Photon analog capture with the simple physics treatment is not tested as indicated by inactivity in label 30 of COLIDP. To test this feature, a CUT:P card with zero weight cutoffs would have to be added to problem 4 or 10 .

- Fluorescent photoelectric photons are not killed by energy importances or weight windows in any of the problems as indicated by inactivity in label 300 of COLIDP.

- Photons from double fluorescence do not make contributions to DXTRAN as indicated in label 90 of DXTRAN and label 90 of TALLYD.

\section{Variance Reduction}

- The VECT card is not used as indicated in EXTRAN, OLDCD1, labels 260, 380 and 400 of CELSRF, and label 110 of NEXTIT. This problem can be corrected by adding the VECT card to problem 6, which uses the exponential transform.

- The PWT card is not tested as indicated by inactivity in label 200 of NEWCRD and label 160 of NEXTIT.

- Some bremsstrahlung biasing options are not tested as indicated by inactivity in labels 110-140 of TTBR. 
- Forced collisions are not used with KCODE calculations, as indicated by inactivity in label 80 of FORCOL.

- The DD card for DXTRAN does not have positive entries as indicated by inactivity in label 220 of DXTRAN.

- the Detector Contribution Card (PD) is not tested on cell cards as indicated by inactivity in label 80 of CELPAR.

\section{Materials}

- The NONU card with entries is never tested as indicated by inactivity in label 330 of NEXTIT, and "DO 330 LOOP" of GETXST.

- The M card GAS, ESTEP, NLIB, PLIB, and ELIB options are not tested as indicated by inactivity in labels 717-728 in NEXTIT, label 40 in RONGE, and labels 175-177 in NXTIT1.

- The VOID card with entries is not tested as indicated by inactivity in label 810 of NEXTIT. Test problem 13 tests the VOID card with cell entries.

- Multiple DRXS libraries used with identical energy bounds are not tested as indicated by inactivity in label 60 of MCRUN.

Data

- The sampling of the energy of an output neutron based on Laws $1,4,5,7,9,11$, $22,24,44$, and 66 is not tested. This omission can be determined by the inactivity of labels $40-50,95-96,160,170-180,190-200,210-220,230-240,242,155$, and 245-252 in the subroutine ACECAS. In addition, labels 180-300 in ACECOL and label 592 of CALCPS are inactive further indicating that law 44 is not used.

- The XS card is not used in any of the input files. The absence of this card can be determined by the inactivity of labels $660-700$ in CHEKIT, label 490 in NEWCRD, labels $780-800$ in NEXTIT and labels $10-60$ in IXSDIR.

- The DATAPATH option in the message block is never tested as indicated by the inactivity of label 60 in EXEMES.

- Only cross-section energy distribution tables that do not have linear-linear interpolation are tested as indicated by the inactivity of labels 40-120 in ACEFCN.

- Type 19 fission is not tested as indicated by the inactivity of the subroutine ACEFPT.

- The calculation of $\bar{\nu}$ using polynomials is not tested as indicated by inactivity in ACENU.

- No problem has energies greater than the cross-section table as indicated by the inactivity of label 70 in ACETBL. 
- Type 2 and 3 cross sections are not tested as indicated by inactivity in labels 80-100 in SREAD.

- Detector contributions from a center-of-mass collision are not tested as indicated by inactivity in label 50 of TALLYD. This feature can be tested by using a different cross-section library.

- The sampling of the energy of an output neutron based on the inelastic law 67 (i.e., ENDF/B-VI law 7) is not tested as indicated by inactivity in label 25 in CALCPS.

\section{Peripherals}

- The VOV option on the DBCN card to get the VOV for all tally bins is untested. This omission can be determined by the inactivity of labels 23-25 in TALSHF and inactivity in TALLYP.

- All timing information is not tested including the FOM as indicated by the inactivity of labels 200-230 and 260 in PRTFCC. This feature is omitted because the 3rd PRDMP entry is negative for the test problems which turns off timing. If timing is turned on, then the tests cannot be exactly compared.

- PTRAC cell, surface, energy cutoff, time cutoff, and angle filters are not tested as indicated by the inactivity of labels $240-245$ in PTRAK and labels $80-100$ of SURSRC.

- PTRAC binary writes are not tested as indicated by the inactivity of labels 70-180 in PTRAK.

- The LOST card is not tested as indicated by inactivity in label 980 of NEXTIT, inactivity in PRLOST, and inactivity in BACKUP.

- The FILES card is not tested as indicated by inactivity in label 1000 of NEXTIT, but this is for user patches only.

- The MPLOT card is not tested as indicated by inactivity in label 1490 of NEXTIT, label 620 of OLDCRD, and label 435 of NXTIT1.

\section{Multigroup}

- MGCOL and MGXSPT are not entered, which indicates that the multigroup adjoint options are not tested.

- Coupled $n / p$ multigroup photon generation is untested as indicated by inactivity in MGCOLN.

- Multigroup analog capture is untested as indicated by inactivity in MGCOLN.

- Multigroup fission is not tested as indicated by inactivity in MGCOLN and MGXSPT.

- Multigroup photons are not tested as indicated by the inactivity of subroutine MGCOLP. 
- Generation of Multigroup importances is not tested as indicated by the inactivity of labels 130-570 in MGIMPS, labels 160-240 in SURFAC, labels 210-250 of PRINV, and inactivity in GMGWW.

- Multigroup heating tallies are not tested as indicated by the inactivity of subroutine MGXSPT and labels 300-310 in TALLY. In addition, Multigroup fission heating is not tested as indicated by inactivity in labels 360-370 in TALLY.

- Multigroup secondary particle production is untested as indicated by the inactivity of subroutine MGXSPT.

- Multigroup criticality is not tested as indicated by the inactivity of labels $100-110$ of COLIDK.

- Multigroup energy bins entered by group number are not tested as indicated by inactivity in labels $120-140$ of STARTP.

\section{Others}

- Continue runs are not tested as indicated by the inactivity of labels $150-250$ of EXEMES, labels 440-530 in IMCN, and inactivity in RUNTPQ.

- Fatal errors and bad trouble errors are not tested as indicated by the inactivity of subroutine EXPIRE.

- If MCNP is restarted because of a machine crash, the code to intercept this crash and restart the clocks is not tested as indicated by inactivity in label 40 of TRNSPT.

- DUMN1, DUMN2, and user files are not used, as indicated by inactivity in labels 30-50 of UFILES.

- The bank never overflows are indicated in labels 20-70 and 130 of BANKIT.

\section{The New Test Set}

The development of the new test set required the testing of the 108 features listed above that were not tested by the MCNP4A test set and the associated libraries. Of these features, the most important features were tested first, and then the other features were tested based on such considerations as the complexity of the required test and the associated running time. The details of how the test problems were modified are given in Appendix A. Twenty features remain untested for various reasons. These are described in the next section.

Compromises were required to keep the size and the running time of the new test problems short. The philosophy of the new test set remains the same as for past MCNP test sets:

MCNP test set philosophy:

The MCNP test set is intended to provide a quick assessment of MCNP correctness on a lat forms where MCNP is supported. The test set is intended to be used every time even 11 smallest modification is made in the development of MCNP to ensure that the new develupment does not affect old code features in unexpected ways. Consequently, the test 
set must be fast so it can be run frequently, and its coverage must be as thorough as possible. Testing is achieved by "tracking" in which each test problem is run only a short time and results are required to exactly match the results of the provided standard MCTAL and OUTP file templates for that problem. Any differences must be completely understood. The test problems are not run to convergence and most answers are statistically meaningless. The test problems are deliberately bizarre and push the code to the limit; they are not reasonable examples of how MCNP problems should be set up.

The MCNP test problems verify that MCNP is running properly. They do not validate the answers provided by the code. Validation is provided separately in benchmark calculations which are run to convergence and com?ared to experiments. Some of these benchmarks are listed in the references. ${ }^{5,6,7,8,9,10,11,12,13}$

\section{A. Remaining Untested MCNP Features}

The new test set now covers all but the following 20 MCNP features.

- All timing information including the FOM is not tested. If timing is turned on, then the tests cannot be exactly compared with the standard MCTAL and OUTP templates because problems run different amounts of time even on the same system.

- User-supplied tallies and sources were not tested, since the test set is designied for the standard MCNP code. In particular, the following user-supplied features were not tested:

- User-supplied sources are not tested, particularly routine SOURCE.

- DUMN1, DUMN2, and user files are untested, particularly routine UFILES.

- User-supplied tallies, particularly routines TALLYX and SRCDX, are not tested.

- Not all aspects of DXTRAN in repeated structures were tested because MCNP4A and all preliminary MCNP4B versions so far are wrong for this combination of features.

- The bank never overflows in any of the test problems. Problems which test this feature would be too slow-running and complicated for a fast and short test set.

- Type 2 and 3 cross sections are not tested. Such a test would be imfossible for a test library portable across different computer platforms.

- Repeated structure tallies in lattices with single entry or triple entry constructs are untested. We could not figure out a simple way to test this.

- A skew transformation of a surface resulting in a GQ general quadratic is not generated in any of the problems. In addition, an orthogonal transformation of a torus is not tested. These are not important cases in most MCNP problems and are difficult to test.

- Overflow of the LAJ array (adding a list of other side cells) is not tested. This feature is untested because it would require the addition of a extremely complex geometry to the test set. 
- A general quadratic that degenerates into a sphere and a torus that completely degenerates into an ellipsoid are not tested. The omission of this option is not an importaut MCNP prcblem.

- LIKE BUT cards that, after expansion, cause lines to spill over to continue lines are not tested. No simple case causing this problem could be devised.

- No criticality source points in the lower level of a lattice are tested. No simple case causing this problem could be devised.

- A unskewed spheroid source is not tested. The omission of this feature does not represent an important MCNP problem.

- Photons from double fluorescence do not make contributions to DXTRAN in any of the test problems. No simple case causing this could be devised.

- Some bremsstrahlung biasing options are not tested.

- Law 5 (the General Evaporation Spectrum) is untested. We were unable to find a data library using this law.

- The calculation of $\bar{\nu}$ using polynomials is not tested. We were unable to find a data library using this law.

- The MPLOT card is not tested, since MCNP compiled without graphics does not include the MPLOT capability.

- Fatal errors and bad trouble errors are not tested since such tests would require the addition of several unrealistic and time-consuming calculations to the test problems.

- If MCNP is restarted because of a machine crash, the code to intercept this crash and restart the clocks is not tested. Addition of such a test would require a hardware failure and is beyond the capabilities of the present tests.

- A high-energy reaction other than fission with energy dependent multiplicity (i.e., Kalbach-87 (law 44) ENDF/B-VI) was not tested. This feature was not tested because a ENDF/B-VI library using this part of law 44 could not be found.

\section{B. New Test Coverage is $97 \%$}

The modifications to the MCNP4A test set and the associated test library were performed and the coverage analysis was applied again to determine the improvements in the new test set. The MCNP4A test set covered $85 \%$ of the active lines of MCNP transport calculations; the new test set increases coverage to $97 \%$. Further, the new set runs as fast as the MCNP4A test set and requires a smaller data library.

All tests were done with MCNP4XK, an intermediate MCNP4B version. This version was used because it tested the MCNP4B features to daie and also tested features that had bugs in MCNP4A. 
The MCNP4XK source code contains 27,892 lines of source code, which includes 6,838 continue and comment lines. Thus, if we consider each set of continued lines as a single line, we can reduce the number of active source lines to 21,054. Now the MCNPXK code also contains 3,785 goto statements, which cannot be traced unless the goto is conditional in nature and the condition is not met. In addition, several lines of the code are provided for errors, plotting, user files, and timing. To obtain an estimate of the number of lines used for these different categories, the number of lines used in the known untested features, not including the four listed previously, were counted. Then this number was subtracted from the total number of lines that were not entered to obtain the number of lines used for untraceable goto statements, errors, plotting, user files, and timing (i.e., 3,214 lines). Thus, the total number of active source code lines used in MCNP was reduced to a final value of 17,840 lines.

The MCNP4A test set covered 15,135 lines out of a possible 17,840 lines (i.e., $85 \%$ ). The new test set covered 17,255 lines (i.e., $97 \%$ ). In addition, the size of the test library has been reduced from $23,346,524$ bytes to $15,142,574$ bytes, and the running speeds of the two libraries are comparable.

\section{MCNP Bugs}

In development of the new MCNP test set, a number of problems were encountered with MCNP4A. Some of these are bugs and some are simply observations.

- DXTRAN in repeated structures could not be tested because of bugs that were encountered.

- MCNP had to be modified so that timing was not printed for continue runs making the test templates not match.

- Multitasking on both the CRAY and distributed processors failed test problem 18 because the old KCODE source overran the new source due to a deliberately poor initial guess of $k_{\text {eff }}=.05$.

- The PLIB identifier cannot be used for the specification of one material if the same zaid appears in the specification of another material. An example of this problem is commented out in new input file INP04.

- Outer parentheses cannot be used on a FM card in the absence of an attenuator (i.e., fm414 ((lll $\left.\left.\begin{array}{lll}1.0 & 1 & 1\end{array}\right)\left(\begin{array}{lll}2.0 & 1 & 2\end{array}\right)\right)$ would cause the code to crash on some systems $)$. This problem can be reproduced by adding back the extra parenthesis on fm414 in problem INP23.

- The use of a UK neutron library (i.e., 29000.02c) will cause MCNP4A to crash on some systems due to the presence of evaluations of the order $1 \times 10^{-99}$. Neutron cross sections are read into MCNP4A as single precision numbers, and numbers of this magnitude cannot be read in using some compilers without specifying double precision formats. The $29000.02 \mathrm{c}$ data in the test library have been adjusted by replacing all occurrences of E-99 with E-30. 
- Multigroup weight windows cannot be generated on some systems due to underflow problems in the smoothing routines. MCNP no longer allows generation of multigroup weight windows less than 1.e-30.

- MODE P E problems do not track exactly the same on different computer platforms due to precision problems. To make them track would require hundreds of changes wherever constants are set.

The other known MCNP4A bugs at the time of this writing (8/1/94) are now listed. These are sorted into three categories: "Significant," "Minor," and "Suspected." Significant bugs silently cause wrong answers. Minor bugs are nuisances which cause the code to crash or perform below expectations but are otherwise harmless. Suspected bugs are bugs that have been reported but are not yet checked out and may merely be user error. A date in parentheses means the bug was corrected in an intermediate MCNP version such as MCNP4XK, an will be fixed in all subsequent versions, and is now tested in the new MCNP test set. Internal X-6 documentation of bug fixes is also noted, such as (X-6:JSH-93-452). Initials in parentheses refer to X-6 personnel who have discovered the problem or are assigned to fix it. When non-X-6 people have been awarded the MCNP $\$ 4$ cash prize for finding a bug, their name and institution are listed.

Significant Bugs:

- Photon transport for hydrogen is wrong for incoherent scatter with detailed physics below $50 \mathrm{KeV}$. (X-6:JSH-93-650). (10/12/93)

- Photon detectors with simple physics are wrong because Form Factors are not turned off. (X-6:JSH-93-656). (10/13/93)

- Contributions to detectors from photon coherent scatter are wrong. (X-6:JSH-93-661). $(10 / 15 / 93)$

- Photon simple physics fails with thick-target bremsstrahlung approximation. $\$ 4$ awarded to Robert Buchl, Rocky Flats (X-6:JSH-93-721) (11/12/93)

- Knock-on electron scatter equation secondary angle wrong. (X-6:HGH-93-745) (12/10/93)

- DXTRAN usually wrong with monodirectional sources. Warning error message added. (X-6:JSH-94-29) 01/24/94

- Distance to DXTRAN sphere must be calculated at level $=0$ or DXTRAN particles not killed. (X-6:JSH-94-101,143) (02/21/94)

- FM xsec is used rather than collision xsec in collisions after FM called with MT=1 or MT=2 (X-6:JSH-94-118) \$4 awarded to Yuji Uenohara, Toshiba Corp., Japan. $(03 / 03 / 94)$

- White boundaries are isotropic in current, not flux. $\$ 4$ awarded to Alfred Hogenbirk, ENL, Netherlands (X-6:JSH-94-379) (06/22/94) 
- TR card operating on a torus silently wrong. Either make N TRCL of cell with torus or TR on toroidal surface fatal, or allow limited translations. When corrected, send $\$ 4$ to Bob Streetman, NIS-12, who reported it 10/2/92

\section{Minor Bugs}

- HP computers crash in continue run if 1st tally has scores 1e-31 below average source weight. $\$ 4$ awarded to Bert Pohl, LLNL (X-6:JSH-93-695) (10/26/93)

- Multitasking lock not turned off for nsr=6,71 Affects UNICOS multitasking efficiency. (RAF) $(12 / 14 / 93)$

- Ptrac option binary files inconsistent with ascii files, causing Sabrina crash. (GWM) $(12 / 14 / 93)$

- Temperatures may be read wrong if data goes past column 80 in XSDIR file. (X6:JSH-94-29) \$4 awarded to Torn Jordan, EMPC (X-6:JSH-94-28) (01/24/94)

- The NONU card fails in a multigroup/adjoint problem. (X-6:JSH-94,29) $(01 / 24 / 94)$

- Multigroup or adjoint problems crash if either the $S(\alpha, \beta)$ thermal treatment or thicktarget bremsstrahlung are turned on. Solution: automatically turn both off. (X-6:JSH94-29) $(1 / 24 / 94)$

- Use of the VOID card with multigroup/adjoint causes a bad trouble exit. Solution: turn off multigroup adjoint if there is a void card. (X-6:JSH-94-29) $(01 / 24 / 94)$

- Nonzero photon importances in cells with zero neutron importances can cause crash. Solution: make fatal error. (X-6:JSH-94-29) (01/24/94)

- Multigroup ZAIDs on XS card cause crash. (X-6:JSH-94-29) Also, there were spurious warning messages. (01/24/94)

- DRXS ZZAAA.nn works but DRXS ZZAAA crashes.(X-6:JSH-94-29) $\$ 4$ awarded to Tom Jordan, EMPC (X-6:JSH-94-28) (01/24/94)

- The exponential transform can sometimes get bad trouble with a zero weight particle. (X-6:JSH-94-29) (01/24/94) \$4 awarded to Tom Jordan, EMPC (X-6:JSH-94-28)

- Near duplicate ZAIDS for photons or electrons can cause a crash. M1 82000.5 82000.01p .5 fails. (X-6:JSH-94-29) (01/24/94)

- Event log can write wrong cell number. (GPE/GWM) (01/28/94)

- Sun Solaris compiler bug fix. Part of output file is missing. (X-6:JSH-94-50) (02/04/94)

- Large values for SDEF WGT keyword can cause FORTRAN overflow errors. TEB (see im4a.61: use 10.d0, not 10) (GWM) (02/11/94) 
- MCNPC.ID NINT function can cause color resolution to be off 1 pixel. $\$ 4$ awarded to Pete Laughton, AECL-CRL, (X-6:JSH-94-78) See line mc.592, line 605. (GWM) $(02 / 14 / 94)$

- Field length printed as zero. (X-6:RAF-94-82, X-6:JSH-94-101) (02/23/94)

- If $\langle c t r l-c\rangle m$ interrupt plot has an error, code returns to transport rather than prompt. (X-6:JSH-94-101) (GWM) (02/24/94)

- MPLOT TFC $P$ command fails. Must reset $f(x)$ array limits. (X-6:RAF-94-82, X$6: J S H-94-101)(02 / 23 / 94)$

- Bill Sailor (LANL, A-3) pointed out that $.90 \mathrm{c}$ fission products cause a crash for E i 1.e-11 on workstations (X-6:JSH-94-95,476) (7/31/94)

- Thermal libraries on XS card cause crash (X-6:JSH-94-122) $\$ 4$ awarded to Heidi McIlwain, AECL-WL, Canada. (03/21/94)

- Multitask versions crash in plot only mode. \$4 Joe Chiaramonte, Schlumberger, CT (X-6:JSH-94-133) (03/23/94)

- MCTAL KCODE plots fail. (X-6:JSH-94-142) \$4 to Nelson Hanan, ANL, IL (03/24/94)

- $\$$ comments fail on column input (\#) entries. $\$ 4$ to G. Bruce Wilkin, AECL-WL (X-6:JSH-94-79) $(02 / 14 / 94)$

- Color graphics bug - random red surface labels with a geometry error. Fix in TEKDVR, tk.55. (GWM) (09/28/93)

- PRDMP 3J 4 entry crashes on continue run.

- An SDEF / repeated structures problem is sdef cel 123 crashes if cell 123 is in $u=1$ and a detector is used. Due to detector one must specify cel $456: 123$ where cell 456 is fill $=1$ or omit cel altogether (losing cell rejection). (RRR)

- We should make fatal if cel=1:2:3, if universes not in ascending order.

- Can't use nJ feature on TR card. (GWM)

- Electron transport fails with WWG.

- An NPS -1 entry fails with KCODE - output not correct (X-6:RAF-90-9).

- Source card combination problem with volume distributed source (SB -21 only causes SP -21 1 and SI 0 1).

- DD entry with continue run crashes if not in initial input.

- Problem with tallying on a surface that has been transformed with use of repeated structures. 
- Incorrect bias through cylindrical window with a spherically symmetric surface source (X-6:ECS-92-204).

- Change NEWCEL error "zero lattice element hit" to 4.2 message "exited finite lattice". Bob Streetman (LANL NIS-12) (N-12-93-477). See suggestions in X-6:GWM-93-597.

- SDEF WGT normalization error with KCODE. Stuart Vessard (LANL-ESH-6) (X6:RAF-93-617). (09/27/93)

- TRCL of a KX (cone) surface with +1 or -1 fails in repeated structures. (RRR)

- MCPLOT BAR command appears to be order sensitive; doesn't work if it is the first command but ok if the last.

- MCPLOT with F8:P,E says photon and F8:N,P says neutron - check units and rel. error.

- Torus geometry problem with large major $\mathrm{R}$ and small minor $\mathbf{R}$ - can get lost particles (Carter $7 / 26 / 91)$.

- Real world lat=1 won't plot lattice surfaces, but lat=2 does.

- Tally on TRCL surface not allowed.

\section{Suspected Bugs}

- Repeated structure tally can produce false DXTRAN error (JFB/GWM)

- Question whether PWT card is used if WWN1:P is used (PWT values in output may be inconsistent).

- DXTRAN with flagged-cell tallies - only flagged if parent particle departs the cell.

- May need -lU77 -lUfor on f77 line of MAKEMCNP for a DECstation 3100. Ray Muzic, Case Western Reserve University, Cleveland $\mathrm{OH}$.

- Electrons can get lost in repeated structures. (GPE)

- SDEF bug with FPOS for RAD and EXT.

- Make F8:N,P,E a warning, not fatal. GWM argues $1 / 19 / 94$ that F8 and mode N should be fatal. $\mathrm{N}$

- Make F6:P,E a warning that energy deposition doesn't include electron contributions.

- Void card with cell entries is not reliable (maybe just with surface source read). 
These problems will probably be corrected in MCNP4B.

Although the "significant" errors do cause wrong answers, they are in very specialized or peculiar cases. In particular, the simple physics treatment bugs occur in regimes where the simple physics treatment should not be used in the first place. None of these bugs is deemed serious enough to warrant replacing MCNP4A.

Unfortunately, the confusion that would be caused by having many MCNP versions used instead of the single MCNP4A version throughout the world makes it a bad idea to attempt correcting the above problems individually. Therefore, the corrections to the above problems will be made only for specified MCNP versions, such as MCNP4A1, available to sponsors only, or MCNP4B, which should be released by RSIC in 1997.

The LANL Radiation Transport Group (X-6) which develops MCNP regrets that it does not have the resources to duplicate and forward copies of the above referenced internal documentation on bugs. Thus access to these documents is restricted to MCNP sponsors only. It is presently the intention of X-6 to integrate the above documentation into any forthcoming MCNP4B documentation.

The large number of "Significant," "Minor," and "Suspected" bugs listed above are not an indication of unreliability on the part of MCNP. Instead, they are a reflection of the scrutiny MCNP receives from its large user base, estimated at several thousand users world wide. The careful documentation and correction of bugs is a reflection of the high priority MCNP places on quality. The publicizing of bugs and the policy of offering cash awards to non-MCNP personnel who identify as much as a misspelled comment statement speaks for the confidence the MCNP development team has in the code

\section{Conclusion}

The MCNP4A test set has been revised. A new brute force methodology for devising the new test set was required because existing software was inadequate. The new test set is as fast as and shorter than the MCNP4A test set.

The new test set increases coverage of MCNP from $85 \%$ to $97 \%$ with 28 problems. The $108 \mathrm{MCNP} 4 \mathrm{~A}$ features or combinations of features not covered by the MCNP4A test set are listed herein along with the remaining 20 ieatures still not covered. The changes in the new test problems from MCNP4A are described in detail.

Development of the new MCNP test set uncovered some code bugs and all known MCNP4A code bugs, minor and suspected as well as significant, are described here.

For years the MCNP development philosophy has been, "Quality, Value, and then Features." Despite the large number of impressive new features put into MCNP in the past few years, quality and value have always been a higher priority. This document and the work it represents is an ongoing part of the MCNP Software Quality Assurance program. It is the intention of the LANL $\mathrm{P}$ diation Transport Group to make MCNP the highest quality general-purpose widely-used Monte Carlo neutron/photon/electron transport code in the world. 


\section{References}

1. J. F. Briesmeister, Ed., "MCNP - A General Monte Carlo N-Particle Transport Code, Version 4A," Los Alamos National Laboratory report, LA-12625-M (1993)

2. R. Kinsey, Compiler, "ENDF-201 ENDF/B Summary Documentation," Brookhaven National Laboratory report BNL-NCS-17541 (ENDF-201) 3rd Edition (ENDF/B-V) (UC-80) (July 1979)

3. P. F. Rose and C. L. Dunford, Editors, "ENDF-102 Data Formats and Procedures for the Evaluated Nuclear Data File ENDF-6," Brookhaven National Laboratory report BNL-NCS-44945 (July 1990)

4. UNICOS ${ }^{R T M}$ Performance Utilities Reference Manual, SR-2040 7.0, Cray Research, Inc. (1992)

5. D. J. Whalen, D. E. Hollowell, and J. S. Hendricks, "MCNP: Photon Benchmark Problems," Los Alamos National Laboratory report LA-12196 (1991)

6. D. J. Whalen, D. A. Cardon, J. L. Uhle, and J. S. Hendricks, "MCNP: Neutron Benchmark Problems," Los Alamos National Laboratory report LA-12212 (1991)

7. J. C. Wagner, J. E. Sisolak, and G. W. McKinney, "MCNP: Criticality Safety Benchmark Problems," Los Alamos National Laboratory report LA-12415 (1992)

8. S. Sitaraman, "MCNP: Light Water Reactor Critical Benchmarks," General Electric Nuclear Energy report NEDO-32028 (1992)

9. C. Crawford and B. M. Palmer, "Validation of MCNP: SPERT-D and BORAX-V fuel," Westinghouse Idaho Nuclear Company, Inc. report WINCO-1112 (1992)

10. C. Crawford and B. M. Palmar, "Validation of MCNP, A Comparison with SCALE, Part 1: Highly Enriched I ium Solutions," Westinghouse Idaho Nuclear Company, Inc. report WINCO-] L

11. C. Crawford and B. M. Palmer, "Validation of MCNP, A Comparison with SCALE, Part 2: Highly Enriched Uranium Metal Systems," Westinghouse Idaho Nuclear Company, Inc. report WINCO-1110 (1992)

12. C. Crawford and B. M. Palmer, "Validation of MCNP, A Comparison with SCALE, Part 3: Highly Enriched Uranium Oxide Systems," Westinghouse Idaho Nuclear Company, Inc. report WINCO-1111 (1992)

13. A. M. Ougouag, C. A. Wemple, G. A. Rubio, and J. M. Ryskamp, "MCNP Analysis of the FOEHN Critical Experiment," Idaho National Engineering Laboratory, EG\&G Idaho, Inc. ORNL/TM-12466 (1993) 


\section{Appendix A: Test Set Modification}

This following presentation of test problem modifications provides a brief description of the original problem along with modifications made to test addition portions of the MCNP code.

\section{A. Problem 1}

This problem represents a simple neutron problem that consists of a biased isotropic point source located at the center of several concentric spheres. This problem was modified by making the following replacements:

\section{Replacements}

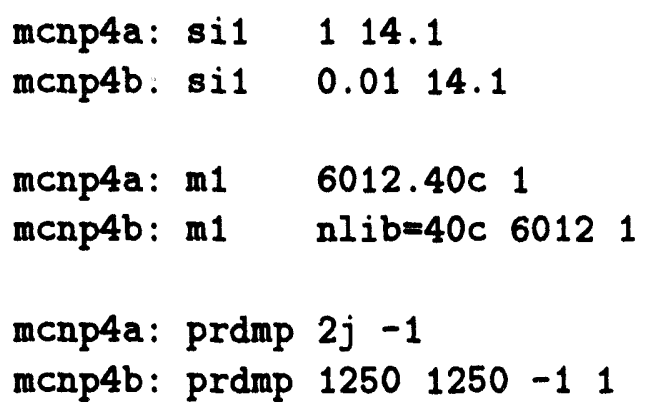

\section{Additions}

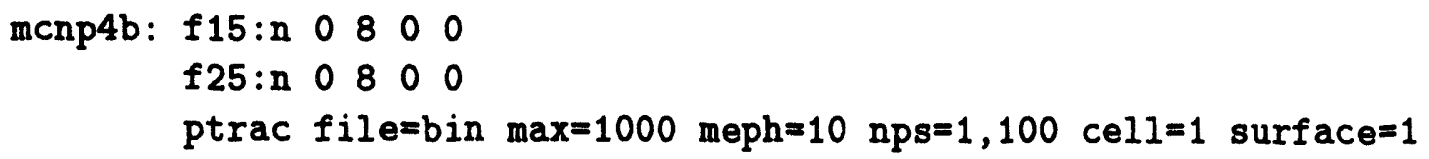

\section{B. Problem 2}

This problem represents a neutron problem that contains a volume source, DXTRAN, and alternate equivalent tallies in a geometry of concentric spheres. The problem was modified by making the following replacements:

\section{Replacements}

$$
\begin{aligned}
& \text { mcnp4a: } 1 \quad 1 \quad-2.45 \quad-1 \quad \$ \text { boron ball with volume source } \\
& \begin{array}{llllllll}
2 & 2 & -2.7 & 1 & -2 & 4 & \$ \text { aluminum shell }
\end{array} \\
& \begin{array}{llllllll}
3 & 2 & -2.7 & 2 & -3 & 4 & \$ \text { aluminum shell }
\end{array} \\
& 40030 \text { outside world } \\
& \begin{array}{llllll}
5 & 2 & -2.7 & 5 & -4 & \$ \text { big aluminum ball to avoid dxtran/tally2 }
\end{array}
\end{aligned}
$$




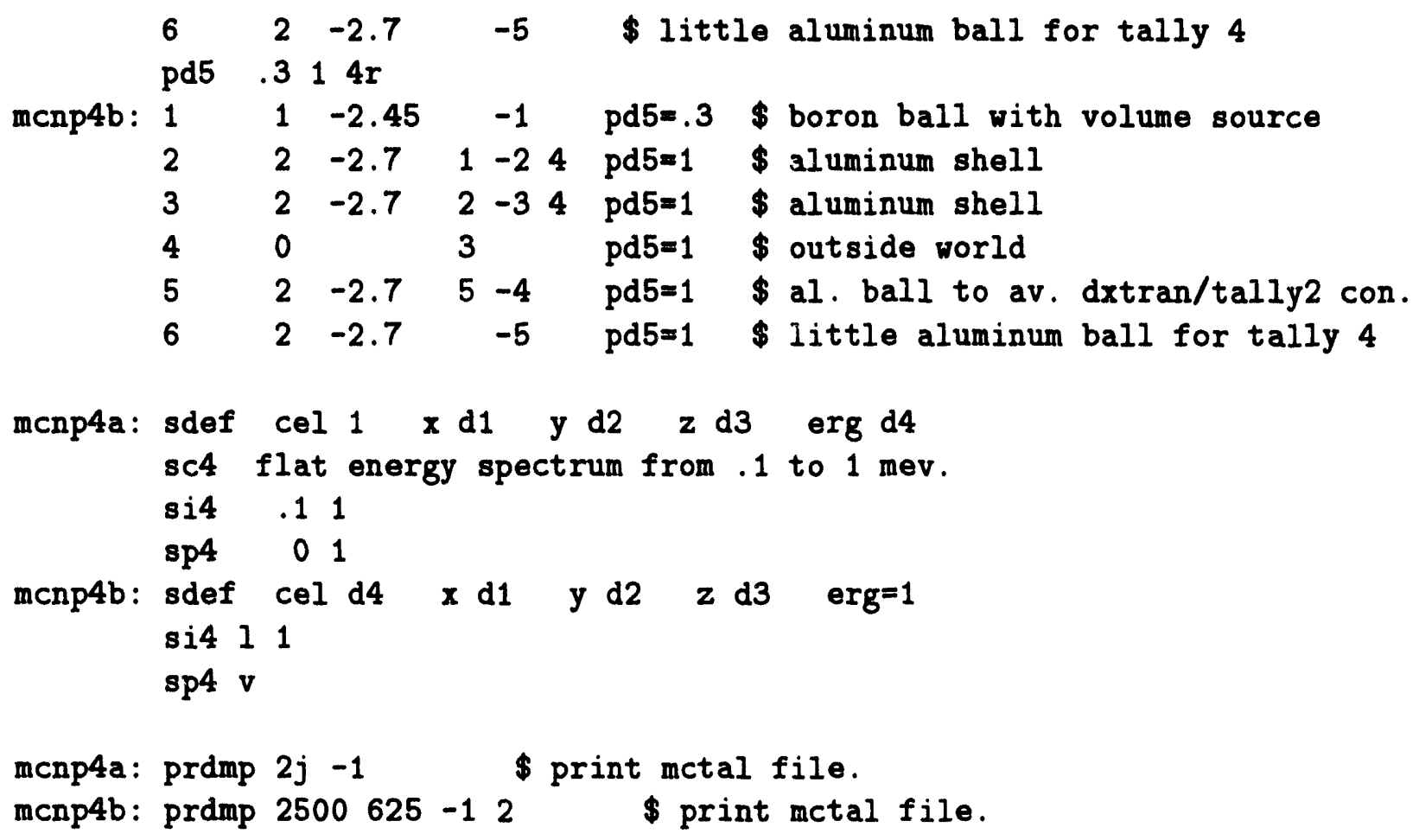

\section{Additions}

mcnp4b: dbcn $14 j 1$

\section{Deletions}

mcnp4a: cut:n $j .001$ \$energy cutoff at $.001 \mathrm{mev}$.

\section{Problem 3}

This problem represents a neutron problem with a sphere and volume source in a box and is designed to test several of the general source features. The modifications to this problem are listed below:

\section{Replacements}

mcnp4a: 14 py 31

menp4b: $14 \quad$ p $0 \quad 310316 \quad 3121 \quad 183127$

menp4a: m1 26000.40 c 1 


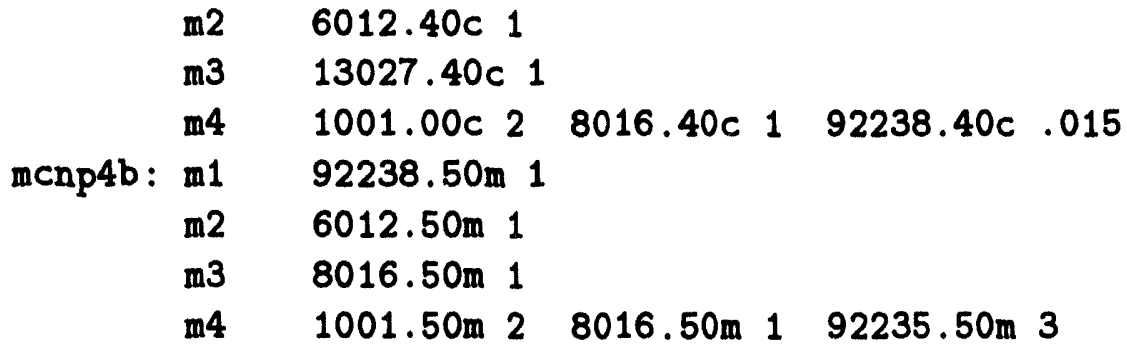

\section{Additions}

mcnp4b: mgopt $f 30$

\section{Problem 4}

This problem represents a photon problem with a point source located at the center of a spherical shell geometry. The modifications to this problem are listed below:

\section{Replacements}

mcnp4a: m1 92000110003

mcnp4b: m1 plib=02p 92000110003 Note: Current version crashes.

mcnp4a: sdef erg $=3 \quad c e l=1$

mcnp4b: sdef erg $=d 1$ cel $=1$ sp1 -4

\section{Additions}

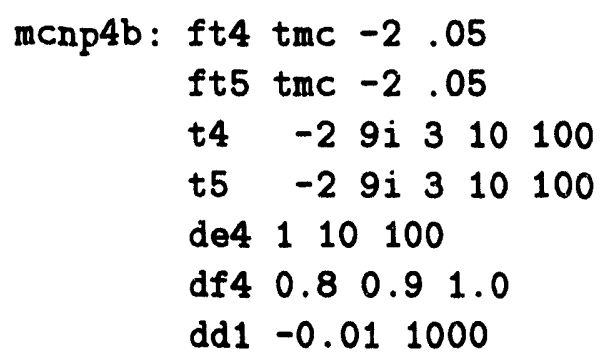




\section{Deletions}

mcnp4a: cut:p $.1 \quad .01 \quad .5 \quad .2 \quad .8$

\section{E. Problem 5}

This problem represents a neutron problem with a volume source in a toroidal tokamak setup. Changes to this problem are outlined below:

\section{Replacements}

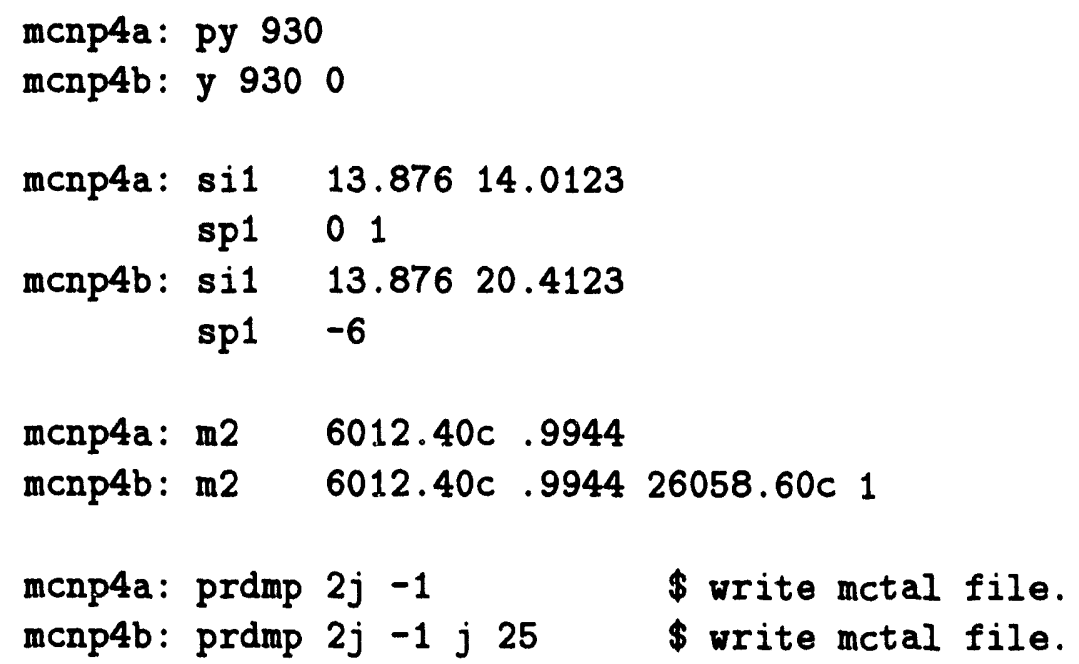

\section{F. Problem 6}

This problem represents a neutron problem with a spherical surface source with position and energy biasing in unison with cutoffs, flagging, and variance reduction techniques. Modifications are outlined below:

\section{Replacements}

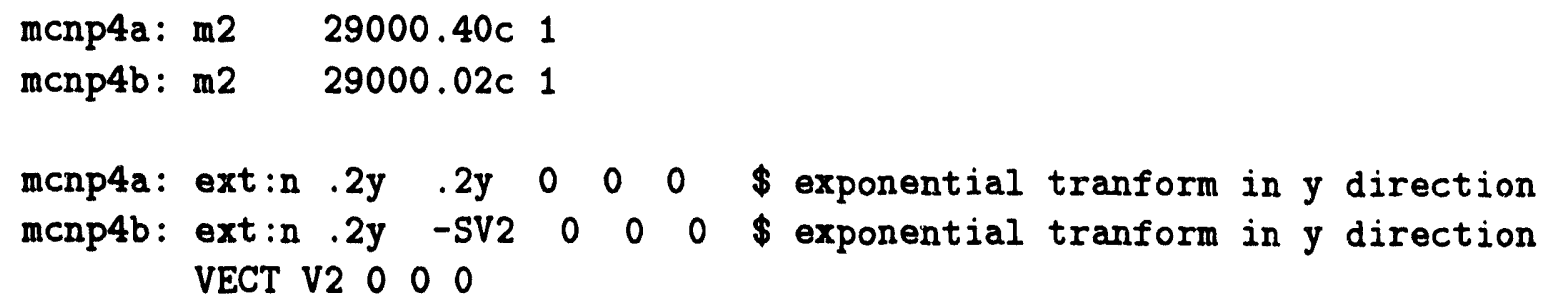




\title{
2. Additions
}

\author{
mcnp4b: $f 14:$ in 2 \\ de14 I.IN 110100 \\ df14 LIN $0.8 \quad 0.9 \quad 1.0$
}

\section{G. Problem 7}

This problem represents a neutron problem with a surface source write on surface number

7. The modifications to this problem are listed below:

\section{Replacements}

$$
\begin{aligned}
& \text { mcnp4a: } 20-12(7: 1:-2) \quad \text { \$empty space } \\
& 3 \quad 5 \quad-5.2 \quad-7 \quad-122 \% 4 \text { \#5 \$ rusty box } \\
& \text { mcnp4b: } 3 \quad 5-5.2 \quad-7 \# 4 \text { \#5 \$6 \$ rusty sphere } \\
& \begin{array}{lllllll}
6 & 7 & -2.7 & -7 & -6 & -3
\end{array} \\
& 85 \quad 5 \quad-5.2 \quad-1 \quad-12 \quad 18 \\
& 9 \quad 5 \quad-5.2-2 \quad-12-19 \\
& \begin{array}{llllll}
10 & 5 & -5.2 & -25 & -12 & 22
\end{array} \\
& \begin{array}{llllll}
11 & 5 & -5.2 & -24 & -12 & 20
\end{array} \\
& 12 \quad 5 \quad-5.2-26-12-23 \\
& \begin{array}{lllllll}
13 & 5 & -5.2 & -27 & -12 & -21
\end{array} \\
& \begin{array}{llllllllll}
17 & 5 & -5.2 & 7 & -18 & 19 & -20 & 21 & -22 & 23
\end{array}
\end{aligned}
$$

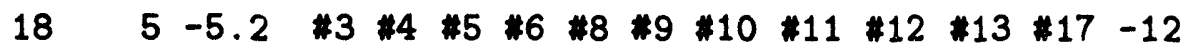

$$
\begin{array}{lllll}
\text { mcnp4a: } & 1 & & c x 15 \\
2 & p x 0 & & \\
3 & p x 10 & & \\
5 & p x 20 & & \\
6 & c / x 2.5 & 0 & 2.5 \\
7 & p x & 40 & & \\
12 & \text { so } 170 & &
\end{array}
$$

menp4b: 3 px -5

5 px 6.25

$6 \quad c / x \quad 0.5 \quad 02.5$

7 so 8

12 so 30

$\begin{array}{lllll}1 & \mathrm{kx} & 18 & 0.22\end{array}$

$2 \quad k / x-18 \quad 1 \quad 0 \quad 0.22$

$25 \quad$ ky $18 \quad 0.22$

$24 \quad \begin{array}{llll}\mathrm{kz} & 18 & 0.22\end{array}$

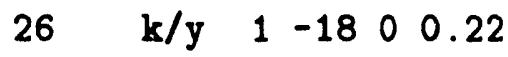


$\begin{array}{lllllll}27 & \mathrm{k} / \mathrm{z} & 1 . & 0 & -18 & 0.22\end{array}$

18 px 17,9

$19 \mathrm{px}-17.9$

20 pz 17.9

$21 \quad$ pz -17.9

22 py 17.9

23 py -17.9

$\begin{array}{rrrrrr}\text { mcnp4a: } & \text { m5 } & 26000.40 c & 1 & 8016.40 c & 1 \\ \text { m6 } & 92235.40 c & 1 & 8016.40 c & 2 \\ \text { mcnp4b: } & \text { m5 } & 26058.60 c & 1 & 8016.40 c & 1 \\ \text { m6 } & 92235.50 \mathrm{~d} & 1 & 8016.40 \mathrm{c} & 2\end{array}$

menp4a: imp:n 00012 r

ssw 7 \$ write the surface source at surface 7

mcnp4b: imp:n $0 \begin{array}{llllllllllll}1 & 1 & 1 & 1 & 1 & 1 & 1 & 1 & 1 & 1 & 1 & 1\end{array}$

ssw 7 sym=1 \$write the surface source at surface 7

mcnp4a: sdef erg d1 cel 4 axs 100 rad d3 ext d2 vec 100 dir d5

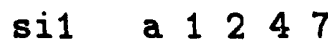

sp1 $0 \begin{array}{llll} & 2 & 1 & 0\end{array}$

mcnp4b: sdef erg fdir d1 cel 4 axs 100 rad d3 ext d2 vec 100 dir d5 $\begin{array}{lllllllllllll}\text { ds1 } & \text { q } & .1 & 21 & .3 & 22 & .6 & 23 & .8 & 25 & 1.24\end{array}$

$\begin{array}{llll}\operatorname{si2} 1 & 0.001247\end{array}$

sp21 02210

sp22 -5

sp23 -6

sp24 -4

si25 a 0.001247

sp25 02210

mcnp4a: si2 101214161820

mcnp4b: si2 $-5 \quad-3-1 \quad 135$

mcnp4a: sd2 706.858 \$aren of surface 7

mcnp4b: sd2 804.22 \$ area of surface 7

\title{
2. Additions
}

\author{
menp4b: sf2 7 \\ cf7 4 \\ lost 2020
}




\section{Deletions}

mcnp4a: sb5 01234

\section{H. Problem 8}

This problem represents a coupled neutron-photon-electron problem with a surface source read from Problem 7 . The changes incorporated into this problem are listed below:

\section{Replacements}

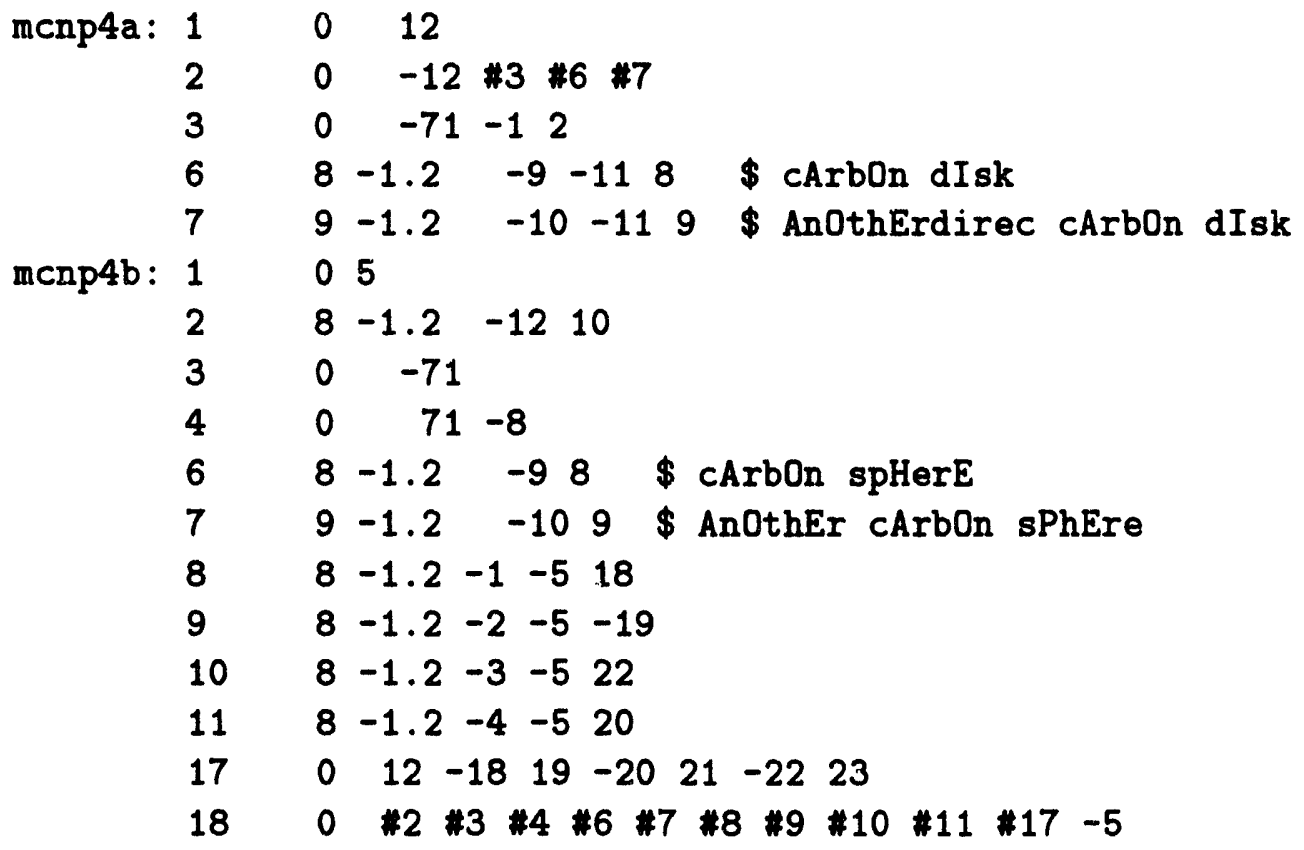

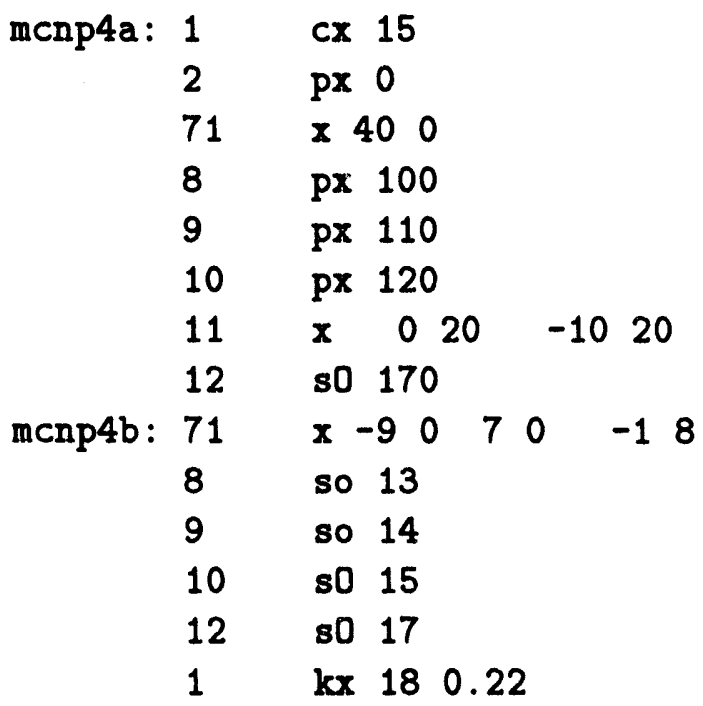




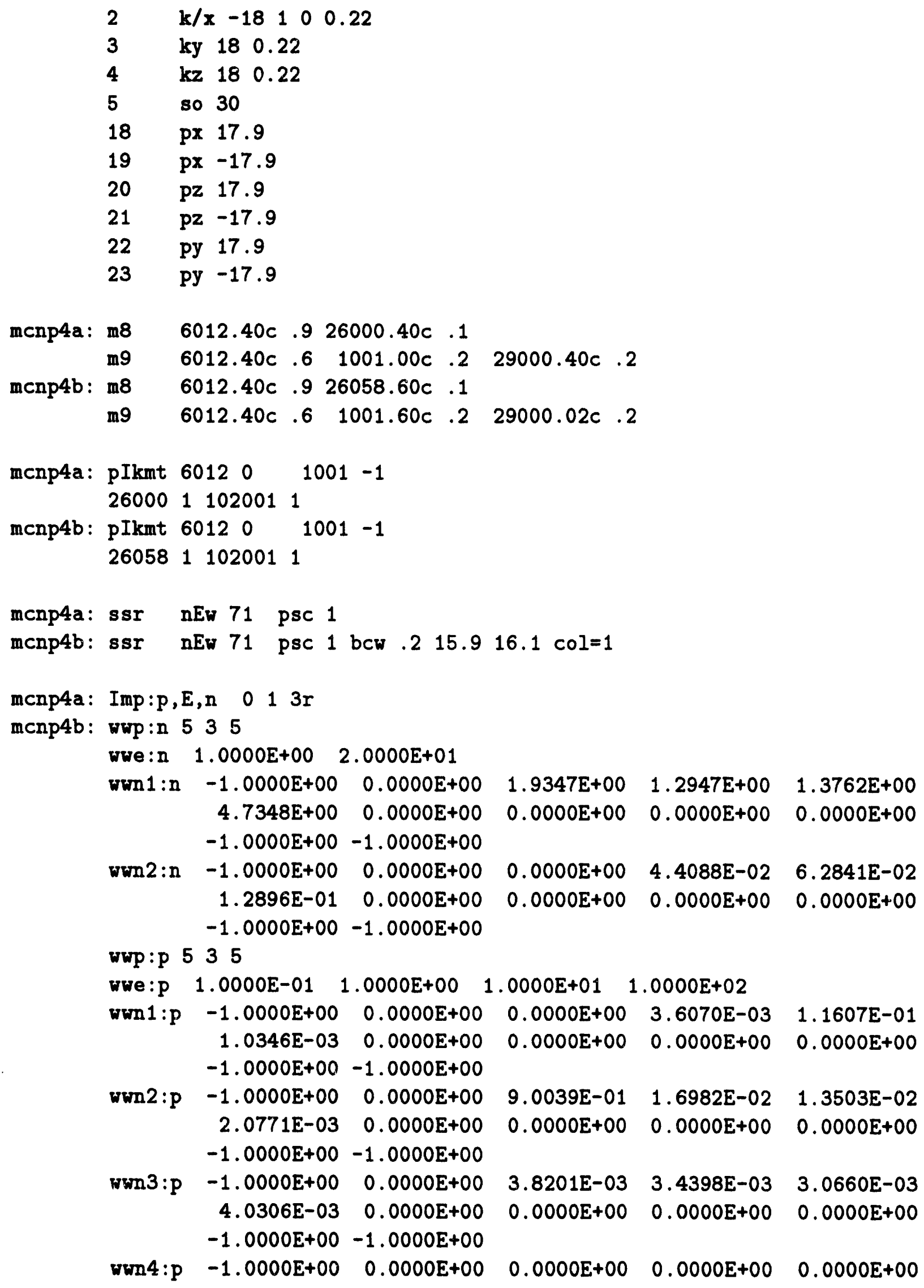




$$
\begin{aligned}
& \begin{array}{lllll}
0.0000 E+00 & 0.0000 E+00 & 0.0000 E+00 & 0.0000 E+00 & 0.0000 E+00
\end{array} \\
& -1.0000 E+00-1.0000 E+00 \\
& \text { wwp:e } 535 \\
& \text { wwe:e } 1.0000 \mathrm{E}-02 \quad 1.0000 \mathrm{E}-01 \quad 1.0000 \mathrm{E}+00 \quad 1.0000 \mathrm{E}+02 \\
& \begin{array}{llllll}
\text { wwn } 1: e & -1.0000 \mathrm{E}+00 & 0.0000 \mathrm{E}+00 & 0.0000 \mathrm{E}+00 & 0.0000 \mathrm{E}+00 & 0.0000 \mathrm{E}+00
\end{array} \\
& \begin{array}{llllll}
0.0000 E+00 & 0.0000 E+00 & 0.0000 E+00 & 0.0000 E+00 & 0.0000 E+00
\end{array} \\
& -1.0000 E+00-1.0000 E+00 \\
& \begin{array}{llllll}
\text { wwn } 2: e & -1.0000 \mathrm{E}+00 & 0.0000 \mathrm{E}+00 & 0.0000 \mathrm{E}+00 & 0.0000 \mathrm{E}+00 & 0.0000 \mathrm{E}+00
\end{array} \\
& \begin{array}{llllll}
0.0000 E+00 & 0.0000 E+00 & 0.0000 E+00 & 0.0000 E+00 & 0.0000 E+00
\end{array} \\
& -1.0000 E+00-1.0000 E+00 \\
& \begin{array}{llllll}
\text { wwn } 3: e & -1.0000 E+00 & 0.0000 E+00 & 0.0000 E+00 & 0.0000 E+00 & 0.0000 E+00
\end{array} \\
& \begin{array}{llllll}
0.0000 \mathrm{E}+00 & 0.0000 \mathrm{E}+00 & 0.0000 \mathrm{E}+00 & 0.0000 \mathrm{E}+00 & 0.0000 \mathrm{E}+00
\end{array} \\
& -1.0000 \mathrm{E}+00-1.0000 \mathrm{E}+00 \\
& \begin{array}{llllll}
\text { wwn } 4: e & -1.0000 E+00 & 0.0000 E+00 & 0.0000 E+00 & 0.0000 E+00 & 0.0000 E+00
\end{array} \\
& \begin{array}{llllll}
0.0000 E+00 & 0.0000 E+00 & 0.0000 E+00 & 0.0000 E+00 & 0.0000 E+00
\end{array} \\
& -1.0000 \mathrm{E}+00-1.0000 \mathrm{E}+00
\end{aligned}
$$

\section{Additions}

mcnp4b: * AREA

101

711

91

81

121

11

21

31

41

51

181

191

201

211

221

231

void 2 


\section{K. Problem 11}

This problem represents a coupled neutron photon problem with a monodirectional source on a plane with a cookie cutter in an intertwined superpretzel geometry. Modification of this problem is outlined below:

\section{Replacements}

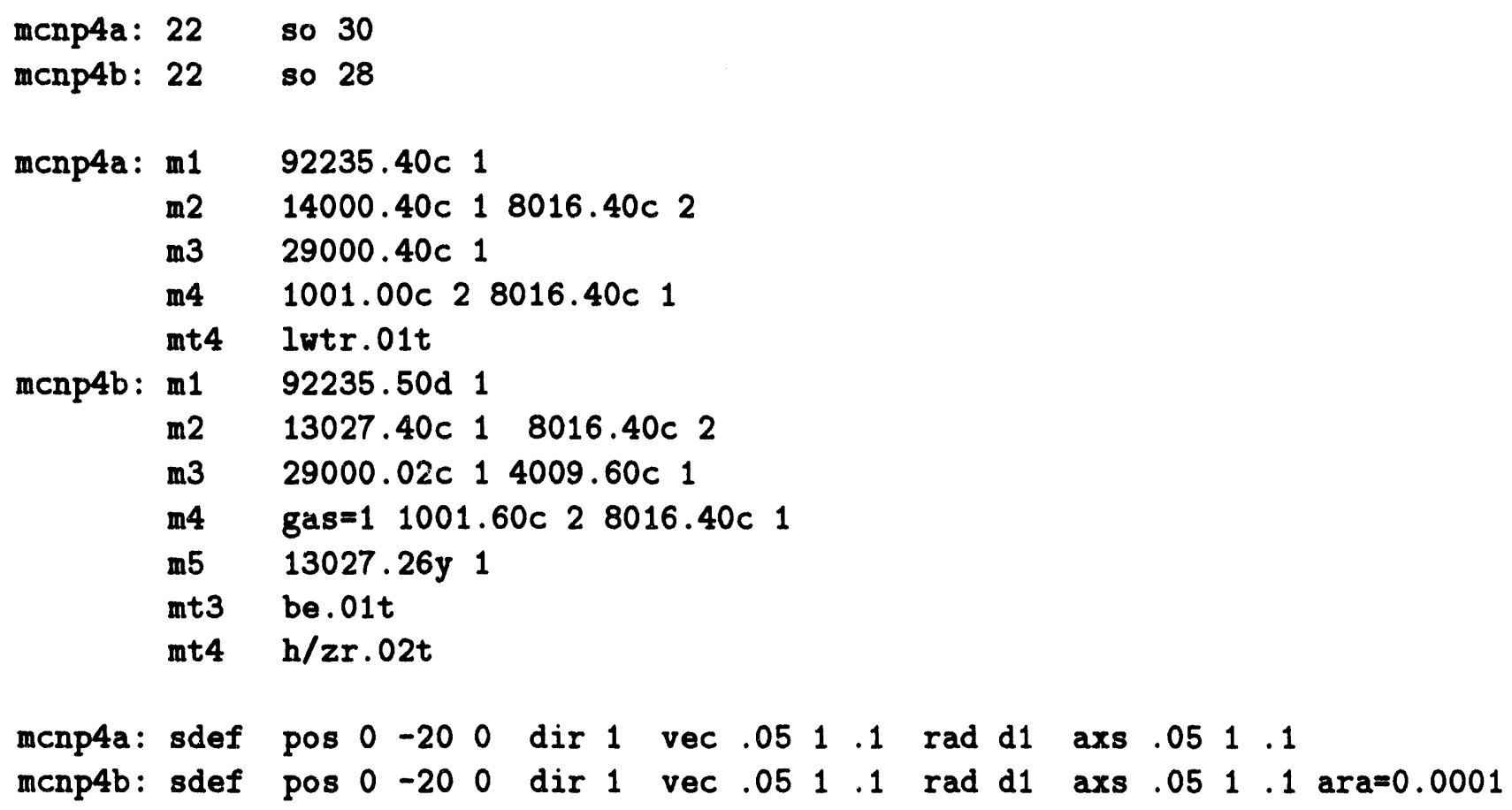

\section{Additions}

mcnp4b: $f m 4 \quad 985103$

$f 14: \mathrm{n} 2$

sd14 (1)

$\operatorname{fm} 14\left(\begin{array}{lll}1 & 2 & -1\end{array}\right)\left(\begin{array}{lll}1 & 2 & -2\end{array}\right)\left(\begin{array}{lll}1 & 2 & -3\end{array}\right)\left(\begin{array}{lll}1 & 2 & -4\end{array}\right)\left(\begin{array}{lll}1 & 2 & 1\end{array}\right)\left(\begin{array}{lll}1 & 4 & 2\end{array}\right)$

$f 24:$ n 2

$\operatorname{sd24}$ (1)

fm24 ((1.0023e-042(102)(51)(52))(1.0-120.000502))

$f 5: n \quad 0 \quad 0 \quad 0 \quad 0$

dxt:n $\begin{array}{lllllll}0 & 0 & 0 & 0.001 & 0.002\end{array}$

$f 15: p \quad 0 \quad 0000$

PWt $\begin{array}{llllll}1 & 1 & -1 & -1 & -1 & -1\end{array}$ 


\section{Problem 12}

Problem 12 is a oil well benchmarking porosity tool model, and for benchmarking reasons this problem was not changed except for use of different cross sections in the test. These cross-section substitutions are listed below:

\section{Replacements}

\begin{tabular}{|c|c|c|c|c|c|}
\hline \multirow[t]{4}{*}{ mcnp4a: } & m1 & $2003.00 \mathrm{c} 1.00000$ & & & \\
\hline & m3 & $1001.00 \mathrm{c} 0.66667$ & $8016.40 c$ & 0.33333 & \\
\hline & m4 & $1001.00 \mathrm{c} 0.15675$ & $6012.40 c$ & 0.15298 & $8016.40 c 0.53730$ \\
\hline & m5 & $1001.00 \mathrm{c} \quad 0.00818$ & $6012.40 c$ & 0.19755 & $8016.40 c 0.59673$ \\
\hline \multirow[t]{4}{*}{ menp4b: } & m1 & $2003.60 \mathrm{c} \quad 1.00000$ & & & \\
\hline & m3 & $1001.60 c \quad 0.66667$ & $8016.40 c$ & 0.33333 & \\
\hline & m4 & $1001.60 \mathrm{c} 0.15675$ & $6012.40 c$ & 0.15298 & $8016.40 \mathrm{c} 0.53730$ \\
\hline & m5 & $1001.60 c \quad 0.00818$ & $6012.40 c$ & 0.19755 & $8016.40 c 0.596$ \\
\hline
\end{tabular}

\section{Problem 13}

This problem is a neutron problem that checks the volume calculator for the rotational symmetry case. The modifications made are listed below:

\section{Additions}

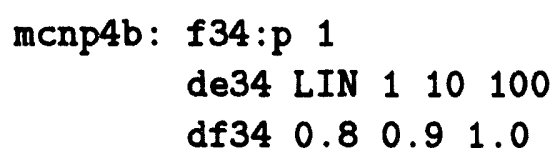

\section{N. Problem 14}

This test represents a neutron problem that tests the general source in repeated structures. The modifications to this problem are listed below:

\section{Replacements}

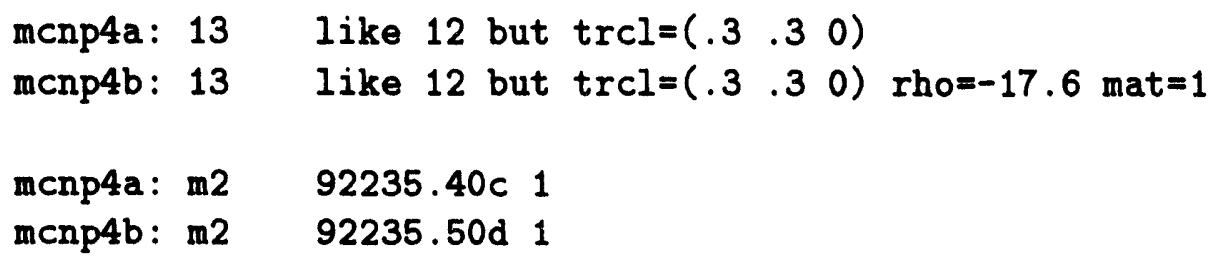




\section{O. Problem 15}

This problem represents a neutron problem test with a filled lattice and a skewed lattice in unison with a general source. The modifications of this problem are listed below:

\section{Replacements}

menp4a: m1 $4009.00 \mathrm{c} 1$

mcnp4b: $m 1 \quad 4009.60 \mathrm{c} 1$

\section{P. Problem 16}

This problem represents a photon problem test with a general source in a lattice. It has been converted to a multigroup problem, and the alterations are outlined below:

\section{Replacements}

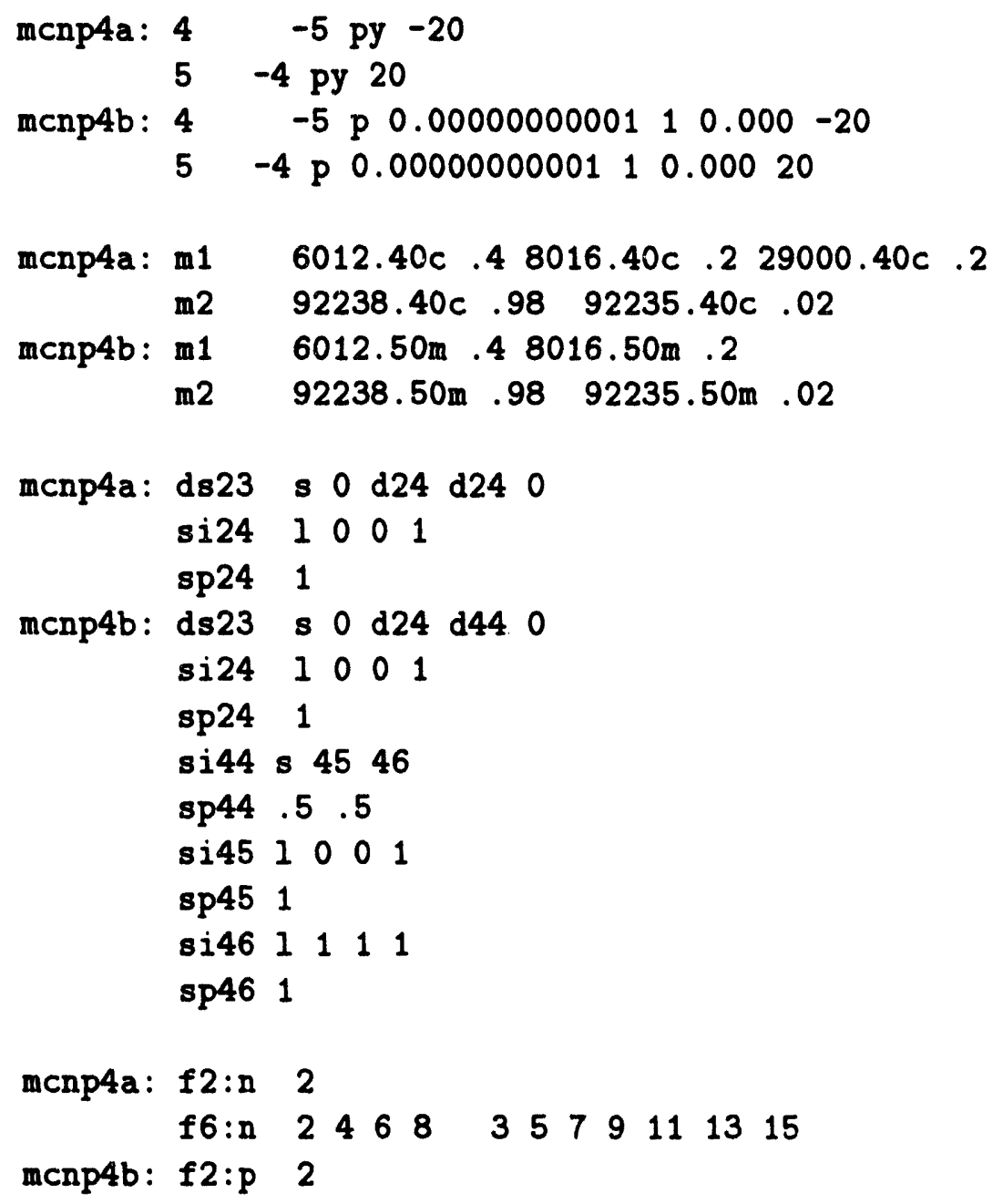




\section{Additions}

mcnp4b: mode $p$

$f 5: p-48-18-58 \quad 0$

dxt:p $\begin{array}{llllllllll}0 & 0 & 0 & 0.05 & 0.1\end{array}$

mgopt $f 12$

phys:p 1

\section{Q. Problem 17}

This problem represents a KCODE problem in a rectangular finite lattice structure. The changes made to this problem are outlined below:

\section{Replacements}

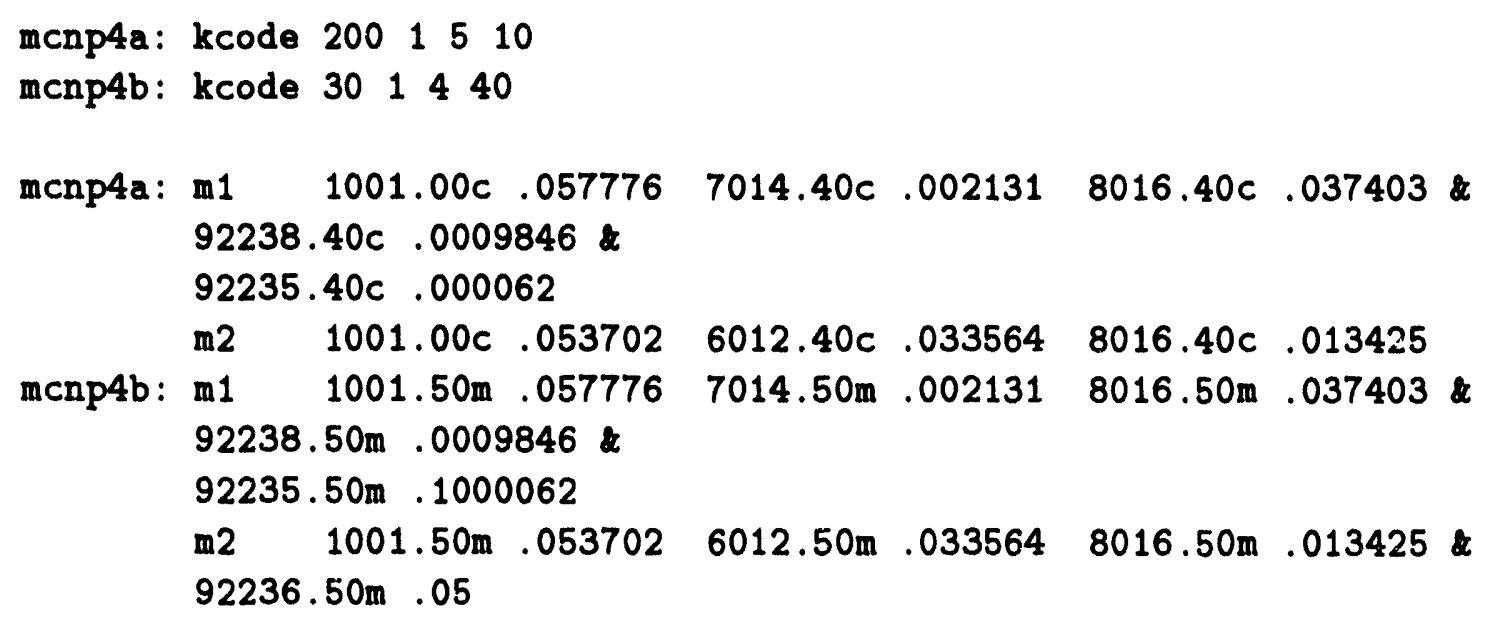

\section{Additions}

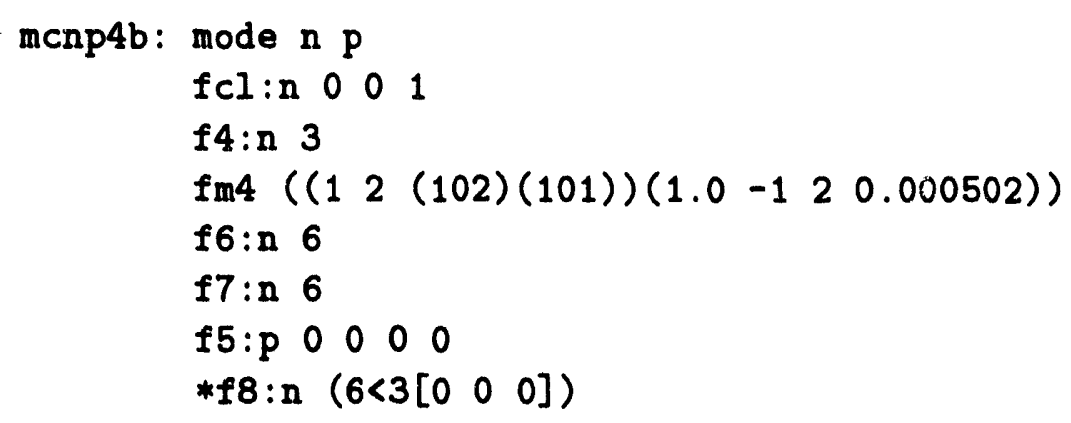


pikmt 1001170141922381

mgopt $f 42$

phys:n 1000.01

\section{R. Problem 18}

This problem represents a neutron KCODE problem in a hexagonal prism lattice. The alterations to this problem are described below:

\section{Replacements}

\begin{tabular}{|c|c|c|c|c|c|c|c|}
\hline \multirow[t]{4}{*}{ mcnp4a: } & $\mathrm{m} 2$ & $92235.40 \mathrm{c}$ & -.70573 & \multirow{2}{*}{$\begin{array}{l}92238.40 c \\
40000.40 c\end{array}$} & -.23821 & \multirow[t]{3}{*}{$7014.40 c$} & \multirow[t]{2}{*}{-.05605} \\
\hline & $\mathrm{m} 6$ & $41093.40 c$ & -.99000 & & -.01000 & & \\
\hline & $\mathbf{m} 7$ & $74000.40 c$ & -.74000 & & & & \\
\hline & $\mathrm{m} 12$ & $1001.00 \mathrm{c}$ & 11002.55 & 8016 & $40 \mathrm{c} 1$ & & \\
\hline \multirow[t]{4}{*}{ mcnp4b: } & $\mathrm{m} 2$ & $92235.50 d$ & -.70573 & \multirow[t]{3}{*}{$92238.40 \mathrm{c}$} & -.23821 & $7014.50 \mathrm{~d}$ & -.05605 \\
\hline & $\mathrm{m} 6$ & $41093.40 c$ & -1.00000 & & & & \\
\hline & $m 7$ & $74000.55 d$ & -.74000 & & & & \\
\hline & $\mathrm{m} 12$ & $1001.60 \mathrm{c}$ & $11002.60 c$ & 8016.4 & c 1 & & \\
\hline
\end{tabular}

mcnp4a: kcode $200 \quad 156$

mcnp4b: kcode $200.05 \quad 56$

\section{Additions}

mcnp4b: $f m 4\left(\begin{array}{lll}1 & 2 & -5\end{array}\right)\left(\begin{array}{lll}1 & 2 & -6\end{array}\right)\left(\begin{array}{lll}1 & 2 & -7\end{array}\right)\left(\begin{array}{lll}1 & 2 & -8\end{array}\right)\left(\begin{array}{lll}1 & 2 & 4001\end{array}\right)\left(\begin{array}{lll}1 & 2 & 102021\end{array}\right)$

\section{S. Problem 19}

This problem represents the multigroup Boltzmann-Fokker-Plank version of electron problem 20. It was modified in the following manner:

\section{Replacements}

$$
\begin{aligned}
& \text { menp4a: } 5 \text { py }-10 \\
& 6 \text { py } 10 \\
& \text { mcnp4b: } 5 \quad 1 p x-10 \\
& 62 \mathrm{px} 10
\end{aligned}
$$




\section{Additions}

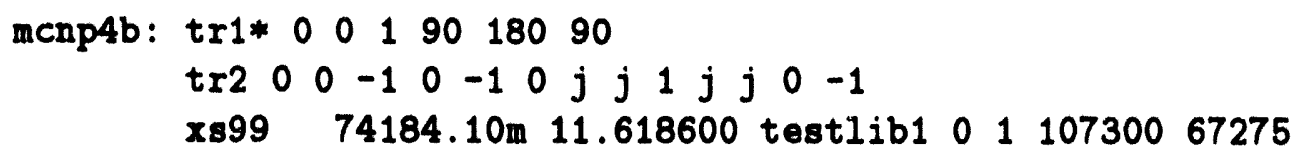

\section{T. Problem 20}

This represents the continuous energy version of the electron problem outlined in problem 19. The modification of this problem is outlined as follows:

\section{Replacements}

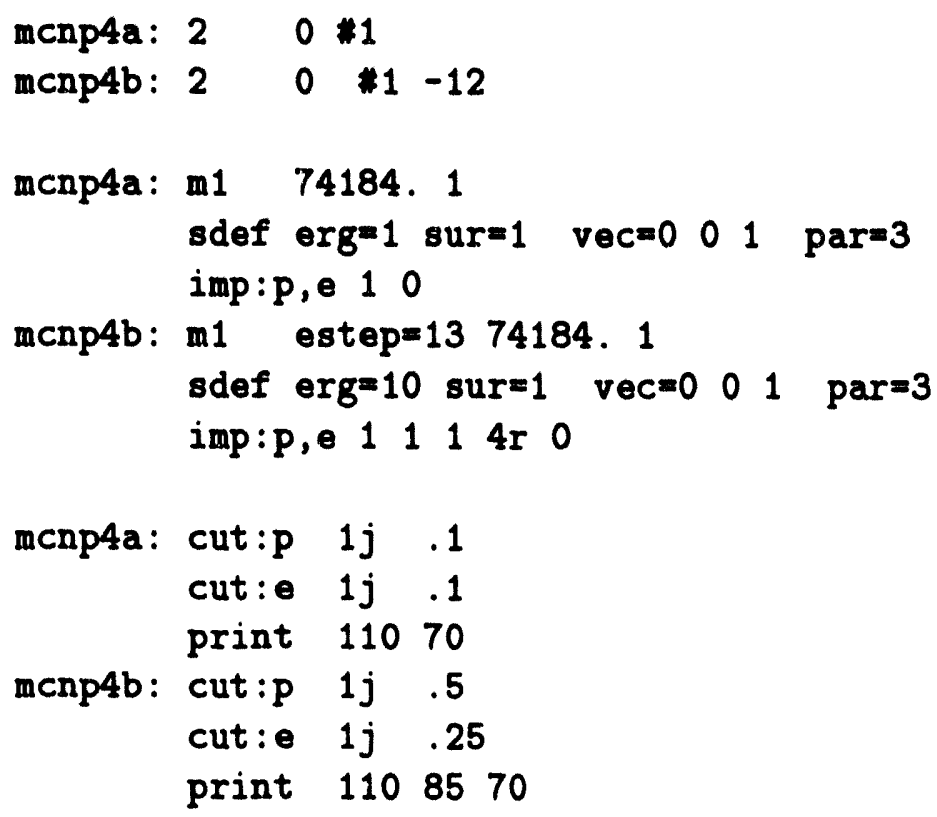

\section{Additions}

$$
\begin{aligned}
& \text { mcnp4b: } 3 \quad 1-19.3 \quad 12-16 \\
& \begin{array}{lllll}
4 & 1 & -19.3 & 16 & -17
\end{array} \\
& \begin{array}{lllll}
5 & 1 & -19.3 & 17 & -18
\end{array} \\
& \begin{array}{llllll}
6 & 0 & 18 & -13 & -14 & 15
\end{array} \\
& \begin{array}{lllllll}
7 & 1 & -19.3 & 13 & -19 & -14 & 15
\end{array} \\
& 80 \text { 19:14:-15 } \\
& \begin{array}{llllll}
12 & 8 & 0.01 & 0.01 & 0 & 15
\end{array} \\
& 13 \text { Cz } 16 \\
& 14 \text { pz } 16
\end{aligned}
$$




$$
\begin{aligned}
& 15 \quad p z-16 \\
& \begin{array}{lllll}
16 & 8 x & 0.01 & 15.2
\end{array} \\
& 17 \text { sy } 0.01 \quad 15.4 \\
& 18 \text { sz } 0.01 \quad 15.6 \\
& 19 \mathrm{c} / \mathrm{z} \quad 0.01 \quad 0 \quad 16.2
\end{aligned}
$$

\section{U. Problem 21}

This problem represents a coupled photon-electron problem that generates a surface source for problem 22. This problem was not modified in relation to the original MCNP4A test set and libraries.

\section{Problem 22}

This problem is a coupled photon electron problem that uses the surface source write from problem 21. The alterations to this problem are outlined below:

\section{Replacements}

menp4a: cut:p $1.0+10 \quad .001-.5-.25$

mcnp4b: cut:p $20 \quad .001-.5-.25$

menp4a: ssr wgt=1

mcnp4b: ssr old $-24124 \quad 224-324 \quad-424$ wgt $=1.5$

\section{W. Problem 23}

This problem represents the forward 80 group electron-photon detector chip problem. The changes made to this problem are outlined in the following section:

\section{Replacements}

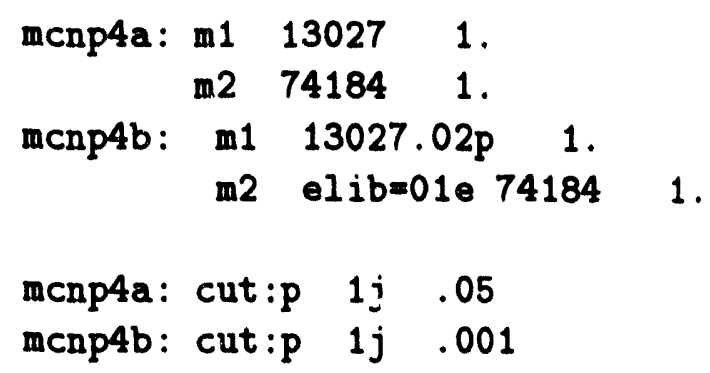




\section{Additions}

mcnp4b: $\operatorname{fm4} 14\left(\left(\begin{array}{llll}1.0 & 1 & 1\end{array}\right)\left(\begin{array}{lll}2.0 & 1 & 2\end{array}\right)\right)$

ft8 Geb 120

dxt:p $00000.001 \quad 0.005$

\section{Problem 24}

This problem represents a KCODE problem in a reflecting lattice without uranium enrichment. The changes to this problem are outlined in the following section:

\section{Replacements}

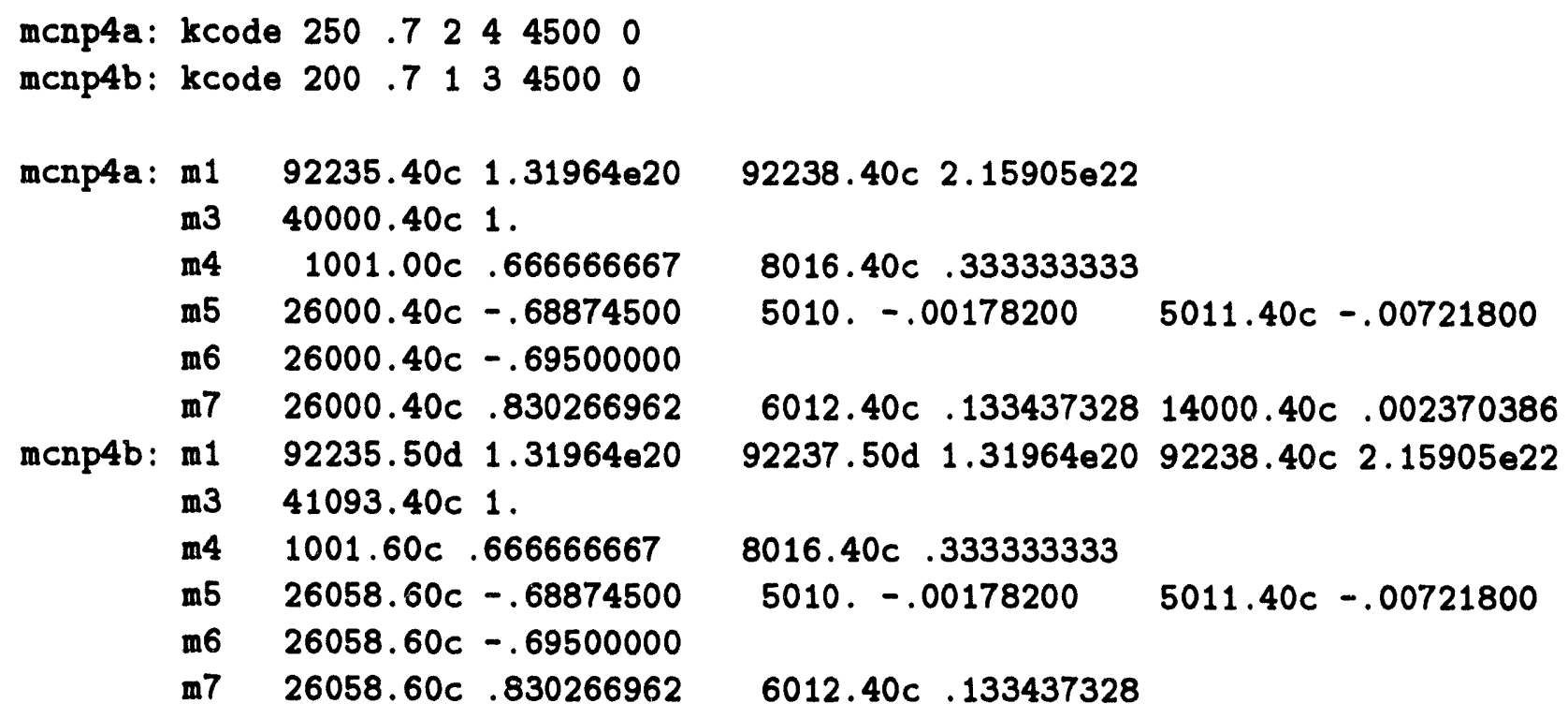

\section{Additions}

nonu $118 \mathrm{r} 0$

\section{Y. Problem 25}

This problem represents a continue run of problem 24 for an additional KCODE cycle. The new input file is listed below:

CONTINUE

$\begin{array}{llll}\text { KCODE } & 250 & .7 & 14\end{array}$ 


\section{Z. Problem 26}

This problem represents a continue run of a surface source write from problem 9 and is listed below:

CONTINUE

kcode 20011055

\section{A. Problem 27}

This problem is a neutron problem that uses the surface source written in problems 9 and 26 as a fission source. The changes to this problem are outlined below:

\section{Replacements}

$\begin{array}{rll}\text { menp4a: } & \text { m1 } & 92235.40 c 1 \\ \text { m2 } & 29000.40 c-1 \\ \text { m3 } & 8016.40 c 17014.40 c 1 \\ \text { menp4b: } 1 & \text { m1 } 92235.50 d 1 \\ \text { m2 } & 29000.02 c-1 \\ \text { m3 } & 8016.40 c 17014.50 d 1\end{array}$

\section{B. Problem 28}

This problem is a new problem in the test set, and it represents a coupled neutron-photon adjoint problem where photons are masquerading as neutrons. The complete input file is listed below:

message: outp=ex5a. out runtpe=ex5a.run

Example 1: Coupled Neutron-Photon Adjoint Problem

C

c

$1 \quad 1-11.35 \quad-1 \quad$ imp: $n=1$

$21-11.35 \quad 1-2 \quad$ imp: $n=1$

$30 \quad 2-4 * 5 \quad$ imp: $n=1$

$40 \quad 4-3 * 5 \quad$ imp: $n=1$

$50 \quad-5 \quad$ imp: $n=1$

$60 \quad 3 \quad$ imp: $n=0$

1 so 20.0

2 so 25.0 


$\begin{array}{llll}3 & \text { so } & 50.0 & \\ 4 & \text { so } & 45.0 & \\ 5 & \text { sz } & 45.0 & 4.999 \\ c & & & \end{array}$

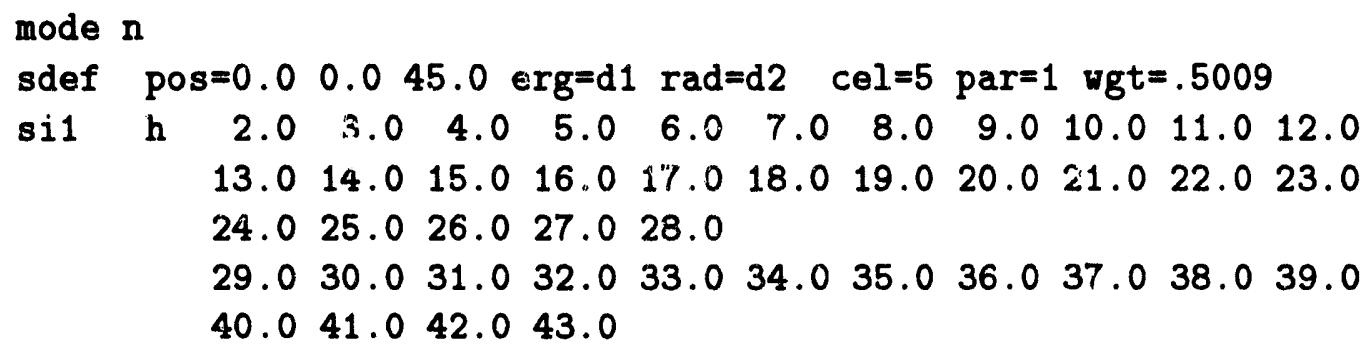




\begin{tabular}{|c|c|c|c|c|c|}
\hline \multirow{3}{*}{ wwn $2: n$} & \multicolumn{5}{|l|}{$-1.0000 E+00$} \\
\hline & $1.6711 E+02$ & $2.1119 E+01$ & $3.4476 E+00$ & $3.3733 E+00$ & $1.6711 \mathrm{E}-01$ \\
\hline & \multicolumn{5}{|c|}{$-1.0000 \mathrm{E}+00$} \\
\hline \multirow[t]{2}{*}{$w w n 3: n$} & $8.0051 E+01$ & $6.5281 \mathrm{E}+00$ & $1.2557 \mathrm{E}+00$ & $1.2311 E+00$ & $8.0051 \mathrm{E}-02$ \\
\hline & \multicolumn{5}{|c|}{$-1.0000 \mathrm{E}+00$} \\
\hline \multirow[t]{2}{*}{ wwn $4: n$} & $5.4069 \mathrm{E}+01$ & $4.9303 E+00$ & $1.2882 \mathrm{E}+00$ & $1.2318 E+00$ & $5.4069 \mathrm{E}-02$ \\
\hline & \multicolumn{5}{|l|}{$-1.0000 E+00$} \\
\hline \multirow[t]{2}{*}{ wwn $5: n$} & $6.2214 \mathrm{E}+01$ & $4.9037 \mathrm{E}+00$ & $1.2843 E+00$ & $1.2425 E+00$ & $6.2214 E-02$ \\
\hline & \multicolumn{5}{|c|}{$-1.0000 E+00$} \\
\hline \multirow[t]{2}{*}{ wwn $6: n$} & $3.84 S 2 E+01$ & $3.4007 E+00$ & $1.4221 \mathrm{E}+00$ & $1.3181 E+00$ & $3.8492 \mathrm{E}-02$ \\
\hline & \multicolumn{5}{|c|}{$-1.0000 \mathrm{E}+00$} \\
\hline \multirow{2}{*}{ wwn $7: n$} & 3.55 & $3.6926 \mathrm{E}+00$ & $1.2577 \mathrm{E}+00$ & $1.2708 \mathrm{E}+00$ & $3.5968 \mathrm{E}-02$ \\
\hline & \multicolumn{5}{|c|}{$-1.0000 E+00$} \\
\hline \multirow[t]{2}{*}{ wwn8:n } & $5.1407 \mathrm{E}+01$ & $3.8684 E+00$ & $1.2702 E+00$ & $1.2185 E+00$ & $5.1407 \mathrm{E}-02$ \\
\hline & \multicolumn{5}{|c|}{$-1.0000 E+00$} \\
\hline \multirow[t]{2}{*}{ won $9: n$} & $3.7975 E+01$ & $4.3719 E+00$ & $1.3182 E+00$ & $1.2814 E+00$ & $3.7975 \mathrm{E}-02$ \\
\hline & \multicolumn{5}{|c|}{$-1.0000 E+00$} \\
\hline \multirow[t]{2}{*}{ wwn $10: n$} & \multirow{2}{*}{\multicolumn{5}{|c|}{$-1.0000 E+00$}} \\
\hline & & & & & \\
\hline \multirow[t]{2}{*}{ wwn $11: n$} & 5.19 & $4.5845 E+00$ & $1.3512 E+00$ & $1.2730 E+00$ & $5.1924 E-02$ \\
\hline & \multicolumn{5}{|l|}{$-1.0000 E+00$} \\
\hline \multirow[t]{2}{*}{ wwn $12: n$} & $4.2609 E+01$ & $4.7344 E+00$ & $1.3720 \mathrm{E}+00$ & $1.2565 E+00$ & $4.2609 \mathrm{E}-02$ \\
\hline & \multicolumn{5}{|l|}{$-1.0000 \mathrm{E}+00$} \\
\hline \multirow[t]{2}{*}{ wwn13:n } & $4.3837 E+01$ & $7.6496 \mathrm{E}+01$ & $7.8580 \mathrm{E}+01$ & $7.0522 E+01$ & $5.2656 \mathrm{E}-01$ \\
\hline & $-1.0000 E+00$ & & & & \\
\hline wwn $14: n$ & 1.0623 & $2.8204 \mathrm{E}+02$ & $7.0076 E+01$ & $6.3159 \mathrm{E}+01$ & $1.0623 E+00$ \\
\hline wwn $15: n$ & 4.20 & $1.7879 E+03$ & $2.3724 E+03$ & $2.3269 E+03$ & $1.8078 \mathrm{E}+01$ \\
\hline & -1.00 & & & & \\
\hline wwn $16: n$ & $4.2043 E+03$ & $3.8991 E+03$ & $2.3724 E+03$ & $2.3269 E+03$ & $1.8078 \mathrm{E}+01$ \\
\hline & $-1.0000 E+00$ & & & & \\
\hline wwn $17: n$ & $4.2043 E+03$ & $2.8901 E+03$ & $2.3724 E+03$ & $2.3269 E+03$ & $1.8078 \mathrm{E}+01$ \\
\hline & $-1.0000 E+00$ & & & & \\
\hline wwn $18: n$ & $4.2043 E+03$ & $5.7802 E+02$ & $1.9167 E+03$ & $2.3269 \mathrm{E}+03$ & $1.8078 \mathrm{E}+01$ \\
\hline & -1.00 & & & & \\
\hline wwn $19: n$ & $3 E+03$ & $9.6139 \mathrm{E}+02$ & $2.3067 \mathrm{E}+03$ & $2.3269 E+03$ & $1.8078 \mathrm{E}+01$ \\
\hline & -1.0 & & & & \\
\hline wen $20: n$ & +03 & $3.8991 E+03$ & $2.3724 E+03$ & $2.3269 \mathrm{E}+03$ & $1.8078 \mathrm{E}+01$ \\
\hline & -1.00 & & & & \\
\hline won21:n & $4.2043 E+03$ & $3.8991 E+03$ & $2.3724 E+03$ & $2.3269 E+03$ & $1.8078 E+01$ \\
\hline & $-1.0000 E+00$ & & & & \\
\hline wwn 22: $n$ & $4.2043 E+03$ & $3.8991 E+03$ & $2.3724 E+03$ & $2.3269 \mathrm{E}+03$ & $1.8078 E+01$ \\
\hline & $-1.0000 E+00$ & & & & \\
\hline $0: n$ & $4.2043 E+03$ & $3.8991 \mathrm{E}+03$ & $2.3724 E+03$ & $2.3269 E+03$ & $1.8078 E+01$ \\
\hline & & & & & \\
\hline
\end{tabular}




\begin{tabular}{|c|c|c|c|c|c|}
\hline \multirow[t]{2}{*}{ wrm $24: n$} & $4.2043 E+03$ & $3.8991 E+03$ & $2.3724 E+03$ & $2.3269 E+03$ & $1.8078 \mathrm{E}+01$ \\
\hline & $-1.0000 E+00$ & & & & \\
\hline \multirow[t]{2}{*}{ wwn $25: n$} & $1.2686 \mathrm{E}+03$ & $1.0659 E+03$ & $8.6645 E+02$ & $8.1562 E+02$ & $6.1601 \mathrm{E}+00$ \\
\hline & $-1.0000 E+00$ & & & & \\
\hline \multirow[t]{2}{*}{ wwn $26: n$} & $2.5373 E+02$ & $2.1319 E+02$ & $1.7329 E+02$ & $1.6312 \mathrm{E}+02$ & 1. $2320 \mathrm{E}+00$ \\
\hline & $-1.0000 E+00$ & & & & \\
\hline \multirow[t]{2}{*}{ wwn $27: n$} & $5.0746 E+01$ & $4.2638 \mathrm{E}+01$ & $3.4658 E+01$ & 3. $2625 E+01$ & $2.4641 \mathrm{E}-01$ \\
\hline & $-1.0000 E+00$ & & & & \\
\hline \multirow[t]{2}{*}{ wwn28:n } & $1.0149 \mathrm{E}+01$ & $8.5275 E+00$ & $6.9316 \mathrm{E}+00$ & $6.5249 E+00$ & $4.9281 E-02$ \\
\hline & $-1.0000 E+00$ & & & & \\
\hline \multirow[t]{2}{*}{ wwn $29: n$} & $2.0298 E+00$ & $1.7055 E+00$ & $1.3863 \mathrm{E}+00$ & $1.3050 \mathrm{E}+00$ & $9.8562 E-03$ \\
\hline & $-1.0000 E+00$ & & & & \\
\hline \multirow[t]{2}{*}{ wwn $30: n$} & $1.8191 E+00$ & $1.6635 \mathrm{E}+00$ & $1.3322 \mathrm{E}+00$ & $1.3783 E+00$ & $1.0550 \mathrm{E}-02$ \\
\hline & $-1.0000 E+00$ & & & & \\
\hline \multirow[t]{2}{*}{ wwn31:n } & $1.4046 \mathrm{E}+00$ & $1.6089 E+00$ & $1.2880 E+00$ & $1.3079 E+00$ & $9.8614 E-03$ \\
\hline & $-1.0000 E+00$ & & & & \\
\hline \multirow[t]{2}{*}{ wwn32:n } & $2.5183 E+00$ & $1.5637 \mathrm{E}+00$ & $1.3618 \mathrm{E}+00$ & $1.3310 \mathrm{E}+00$ & $1.0716 \mathrm{E}-02$ \\
\hline & $-1.0000 E+00$ & & & & \\
\hline \multirow[t]{2}{*}{ wwn33:n } & $1.5070 \mathrm{E}+00$ & $1.8127 \mathrm{E}+00$ & $1.3937 E+00$ & $1.3336 \mathrm{E}+00$ & $1.0264 E-02$ \\
\hline & $-1.0000 \mathrm{E}+00$ & & & & \\
\hline \multirow[t]{2}{*}{ wwn $34: n$} & $2.4316 \mathrm{E}+00$ & $1.8490 E+00$ & $1.3110 E+00$ & $1.3272 \mathrm{E}+00$ & $1.0109 \mathrm{E}-02$ \\
\hline & $-1.0000 E+00$ & & & & \\
\hline \multirow[t]{2}{*}{ wwn $35: n$} & $1.5357 \mathrm{E}+00$ & $1.5518 \mathrm{E}+00$ & 1. 2595E+00 & 1. $2655 \mathrm{E}+00$ & $9.7192 E-03$ \\
\hline & $-1.0000 E+00$ & & & & \\
\hline \multirow[t]{2}{*}{ whn36:n } & $1.6220 \mathrm{E}+00$ & $1.5526 \mathrm{E}+00$ & $1.2179 E+00$ & $1.2584 \mathrm{E}+00$ & $9.9295 E-03$ \\
\hline & $-1.0000 \mathrm{E}+00$ & & & & \\
\hline \multirow[t]{2}{*}{ พพน $37: n$} & $1.6606 \mathrm{E}+00$ & $1.8820 E+00$ & $1.3317 \mathrm{E}+00$ & $1.2569 E+00$ & $1.0224 \mathrm{E}-02$ \\
\hline & $-1.0000 E+00$ & & & & \\
\hline \multirow[t]{2}{*}{ พนก $38: n$} & $1.2651 \mathrm{E}+00$ & $1.1517 \mathrm{E}+00$ & 1. $2199 E+00$ & 1. $2742 E+00$ & $1.0539 E-02$ \\
\hline & $-1.0000 E+00$ & & & & \\
\hline \multirow[t]{2}{*}{ whn39:n } & $9.9115 E-01$ & $1.2620 E+00$ & $1.2497 E+00$ & $1.1964 E+00$ & $9.9027 \mathrm{E}-03$ \\
\hline & $-1.0000 E+00$ & & & & \\
\hline \multirow[t]{2}{*}{ wwn $40: n$} & $9.9611 \mathrm{E}-01$ & $1.3924 \mathrm{E}+00$ & $1.1944 E+00$ & $1.2062 \mathrm{E}+00$ & $9.9623 E-03$ \\
\hline & $-1.0000 E+00$ & & & & \\
\hline \multirow[t]{2}{*}{ ww4 $41: n$} & $1.0301 E+00$ & $1.1601 \mathrm{E}+00$ & $1.1090 \mathrm{E}+00$ & $1.2091 E+00$ & $1.0242 E-02$ \\
\hline & $-1.0000 E+00$ & & & & \\
\hline \multirow[t]{2}{*}{ wwn $42: n$} & $9.4191 E-01$ & $1.0000 E+00$ & $1.1647 \mathrm{E}+00$ & $1.2669 E+00$ & $1.0684 E-02$ \\
\hline & $-1.0000 E+00$ & & & & \\
\hline
\end{tabular}



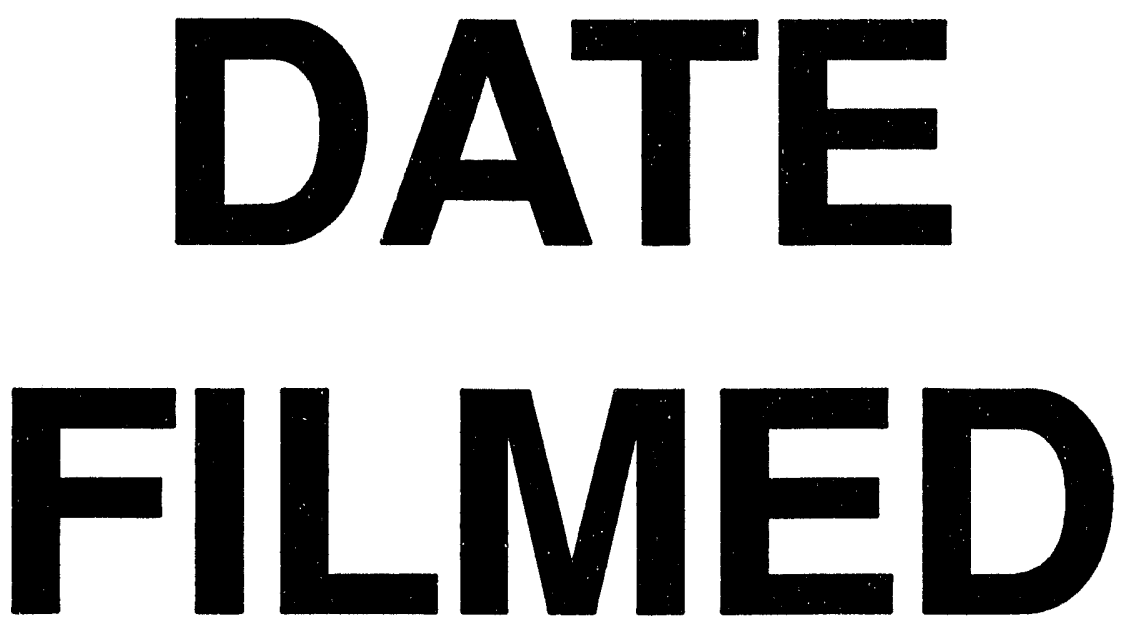

$10 / 5 / 94$
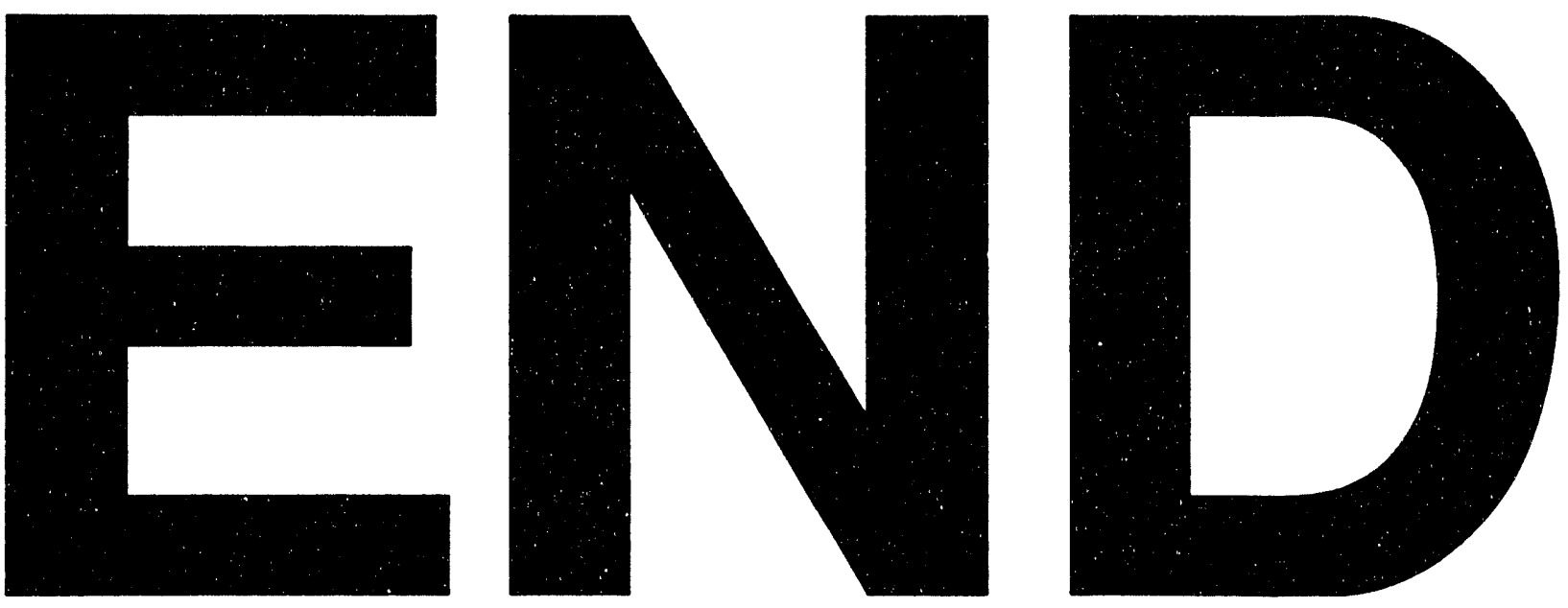
